\title{
Annihilation signals from asymmetric dark matter
}

\author{
Edward Hardy, ${ }^{a}$ Robert Lasenby ${ }^{a}$ and James Unwin ${ }^{b}$ \\ ${ }^{a}$ Rudolf Peierls Centre for Theoretical Physics, University of Oxford, \\ 1 Keble Road, Oxford, OX1 3NP, U.K. \\ ${ }^{b}$ Department of Physics, University of Notre Dame, \\ 225 Nieuwland Science Hall, Notre Dame, IN 46556, U.S.A. \\ E-mail: e.hardy12@physics.ox.ac.uk, Robert.Lasenby@physics.ox.ac.uk, \\ james. unwin@nd. edu
}

ABSTRACT: In the simplest models of asymmetric dark matter (ADM) annihilation signals are not expected, since the DM is non-self-conjugate and the relic density of anti-DM is negligible. We investigate a new class of models in which a symmetric DM component, in the 'low-mass' $1-10 \mathrm{GeV}$ regime favoured for linking the DM and baryon asymmetries, is repopulated through decays. We find that, in models without significant velocity dependence of the annihilation cross section, observational constraints generally force these decays to be (cosmologically) slow. These late decays can give rise to gamma-ray signal morphologies differing from usual annihilation profiles. A distinctive feature of such models is that signals may be absent from dwarf spheroidal galaxies.

Keywords: Cosmology of Theories beyond the SM, Beyond Standard Model

ArXiv EPRINT: 1402.4500 


\section{Contents}

1 Introduction 1

2 Regeneration from models with two dark asymmetric species 4

3 General constraints and signals $\quad 6$

$3.1 \quad B^{\prime}-L^{\prime}$ mass splitting and $\overline{L^{\prime}}$ distribution $\quad 7$

$\begin{array}{lll}3.2 & \text { Direct and indirect detection } & 10\end{array}$

$\begin{array}{lll}3.3 & \text { Annihilation via } L^{\prime} \overline{L^{\prime}} \rightarrow V V & 12\end{array}$

3.4 Annihilation via $L^{\prime} \overline{L^{\prime}} \rightarrow \phi \rightarrow \mathrm{SM} \quad 14$

$\begin{array}{lll}3.5 & \text { Tentative signals and morphology } & 15\end{array}$

4 Model building $\quad 16$

$\begin{array}{lll}\text { 4.1 An implementation with fundamental matter } & 17\end{array}$

$\begin{array}{ll}4.2 \text { Comments on composite-type models } & 19\end{array}$

$\begin{array}{llr}5 & \text { Conclusion } & 20\end{array}$

$\begin{array}{ll}\text { A Rates of } B^{\prime} \text { and } L^{\prime} \text { violating processes } & 21\end{array}$

B $\quad B^{\prime}-L^{\prime}$ mass splitting and $\overline{L^{\prime}}$ injection velocity 23

C Calculating the Galactic $\overline{L^{\prime}}$ distribution $\quad 24$

\section{Introduction}

Asymmetric dark matter $(\mathrm{ADM})$ provides a well motivated framework for light DM and is an intriguing alternative to the usual WIMP scenario. In such models the DM, which we denote here $B^{\prime}$, has an (approximately) conserved quantum number (which we also call $B^{\prime}$ ). The relic density is determined by a particle-antiparticle asymmetry between $B^{\prime}$ and $\overline{B^{\prime}}$, in direct analogy to baryons [1-3]. If the DM has a similar mass to the proton $m_{B^{\prime}} \sim m_{p}$ and the hidden and visible sectors are connected via portal operators which violate $B, L$ and $B^{\prime}$, but conserve some linear combination, then this can explain the cosmological coincidence $\Omega_{\mathrm{DM}} \sim 5 \Omega_{B}$. In contrast, the accidental proximity of $\Omega_{\mathrm{DM}}$ and $\Omega_{B}$ in conventional DM scenarios seems unreasonable given that the relic density of DM is determined by freeze-out, whereas the baryon density is set by CP-violating decays of out-of-equilibrium states and these two mechanisms are typically unrelated.

While we shall not specify the UV physics that produces these particle asymmetries, there are several mechanisms that can cogenerate equal magnitudes for $\eta_{B^{\prime}}, \eta_{B}$ and/or $\eta_{L}$ (see e.g. [4-8]). Alternatively, an asymmetry could be generated in a single quantum 
number and subsequently shared via processes that violate the individual global symmetries, leading to comparable asymmetries. For the case $\eta_{B^{\prime}} \simeq \eta_{B}$, to account for the DM relic density one requires the DM mass to be around $5 \mathrm{GeV}$. Inefficient sharing, or bias generation, of the DM and baryon asymmetries may easily lead to $\mathcal{O}(1)$ deviations between $\eta_{B^{\prime}}$ and $\eta_{B}$ and hence the DM mass could reasonably lie in a relatively large window $0.1-100 \mathrm{GeV}$. However, arguably the most natural mass region is $1-10 \mathrm{GeV}$.

Indirect detection signals of DM can arise if the DM decays or annihilates producing cosmic rays containing high energy photons, electrons, positrons or antibaryons. Usually, the event density associated to signals of decaying DM depends linearly on the DM density $n_{\mathrm{DM}}$, whereas for annihilating $\mathrm{DM}$ the signal has an $n_{\mathrm{DM}}^{2}$ dependence. If astrophysical gamma-ray signals from DM were detected, then their profile on the sky would let us determine which process produced them, e.g. $[9,10]$ (the propagation of charged cosmic rays is affected by galactic magnetic fields, so a signal in these channels would not be so helpful). Whilst decay signals of ADM can readily arise, see e.g. [11], in general, we do not expect late-time annihilation signals from ADM, since the symmetric component is assumed to have annihilated early on. However, if the symmetric component is later 'regenerated', this may give rise to annihilation signals. Previous proposals along these lines include slow DM-antiDM oscillations [12-15], and intermediate-time decays (in the higher DM-mass regime, [16]). Alternatively, there are scenarios in which an asymmetry plays a role in determining the DM relic density, but the DM itself is not asymmetric [1719]. In this paper, we investigate the prospect of regenerating a symmetric component via decays in the low DM-mass regime.

A challenge in building viable models of ADM is obtaining a high enough annihilation cross section so that the relic density is set by the asymmetry and not by a frozen-out symmetric DM component. For this to occur the cosmologically stable states in the dark sector typically must have annihilation cross sections some factor larger than the thermal value $\left(\sim 3 \times 10^{-26} \mathrm{~cm}^{3} \mathrm{~s}^{-1}\right)$. Experimental limits on this scenario arise from a variety of sources. Direct searches rule out large regions of parameter space where the interaction with the visible sector is through heavy portal states that can be parameterised as effective operators, see e.g. [20-22, 27], ${ }^{1}$ although viable models remain if the mediating states are light. Perturbativity also often limits the cross sections that can be obtained in viable models. Additionally, indirect detection constraints are limiting in some regions of parameter space.

Generally, the strongest astrophysical constraints on the DM annihilation cross section (if the population is entirely symmetric) are close to or below the lower cross-section bound from the ADM relic annihilation constraint. In particular, if a sizeable symmetric component is regenerated before the recombination era, then the limits on the annihilation cross section from CMB observations [29-32] are typically only a small factor above the thermal freeze-out value, for DM masses $\lesssim 20 \mathrm{GeV}$. Since the CMB limits are mostly a function of energy injection, rather than the particular annihilation products, and are not subject to uncertainties regarding DM distribution, they leave very little parameter space for early

\footnotetext{
${ }^{1}$ For contact operators with preferential coupling to specific SM states, such as neutrinos [28], muons, or taus, some of the tension with experimental constraints can be alleviated.
} 
decays, for annihilation cross-sections large enough to meet the ADM relic annihilation requirements.

One manner of circumventing these constraints is for the annihilation cross section at late times to be suppressed relative to earlier times, e.g. via velocity-suppression. If the symmetric population is regenerated after freeze-out, but early relative to astrophysical timescales, then the phenomenology of this scenario is much like symmetric DM with a velocity-suppressed cross section, although with the annihilation rate decoupled from the thermal value. An alternative possibility, with distinctive phenomenology, is the case in which the DM remains predominantly asymmetric up to the present time (or at least through the recombination era), with only a small symmetric component regenerated. If the decay timescale is significantly longer than the time at recombination, then CMB constraints are significantly weakened, which can open the possibility of present-day annihilation signals without corresponding CMB signals. Another interesting feature of regenerating the symmetric component through late decays, which we will discuss in some detail, is that its density profile may be modified significantly by the velocity kick from the decay process. Obtaining a sufficiently small velocity kick to keep the regenerated population concentrated in galaxies motivates DM models with a small mass splitting between different components. For these models, the annihilation profile may be different to those arising from conventional models, and could be entirely absent from systems with low escape velocities such as dwarf spheroidal galaxies.

As mentioned, coupling ADM to the Standard Model (SM) without violating direct search constraints requires some model-building, and we consider two scenarios in detail. In one case the annihilation portal to the SM is through a pseudoscalar; this alleviates the direct detection constraints, as the DM-proton scattering cross section is significantly suppressed. If the pseudoscalar mass is just above the value needed for an s-channel annihilation resonance, then annihilations during the freeze-out process (when temperatures are high) are enhanced relative to annihilations at later times. This is essentially an example of the velocity dependence discussed above. The second scenario we consider is where annihilation occurs to two on-shell hidden sector vector bosons, which decay relatively slowly to SM states. The small coupling of the vectors to the SM suppresses direct search constraints, and the rate at which we regenerate the symmetric component controls the strength of CMB and late-time annihilation constraints. These models act as a proof of principle that annihilation signals can be generated from certain models of ADM, but the constraints that we discuss are more generally relevant to other models of annihilating $\mathrm{DM}$ in the low mass region. The observation of signals in this mass region may suggest that the DM relic density is set by an asymmetry, and that the hidden sector dynamics is correspondingly more complicated than often assumed.

Turning to the structure of the paper, in section 2 we begin by discussing an example class of models that regenerates a symmetric component through decays. Subsequently, in section 3 we explore the constraints on models of annihilating ADM and identify the parameter space for which successful models can be constructed. Additionally, we study the modification of signal morphologies due to the decays. Section 4 explores the hidden sector model-building necessary to satisfy the constraints obtained. 


\section{Regeneration from models with two dark asymmetric species}

To construct a model with late time decays, regenerating a symmetric component of DM, requires a more complicated hidden sector. By simple analogy to the complexity of the visible sector it is quite conceivable that the hidden sector consists of multiple states and approximately conserved global currents. As a model building example we consider a particular setup, but the constraints and possible signals we find in section 3 are more generally applicable.

Let us suppose that two states $B^{\prime}, L^{\prime}$ in the hidden sector carry (approximately) conserved quantum numbers which we suggestively call dark baryon number $B^{\prime}$ and dark lepton number $L^{\prime}$. In the SM, baryon and lepton number are approximately conserved; EW instantons and sphalerons, and GUT scale physics do not respect these global symmetries, however these processes still conserve the combination $B-L$. In direct analogy, we propose that both $B^{\prime}$ and $L^{\prime}$ are accidental symmetries of the low energy theory and are violated at some intermediate scale, however the combination $B^{\prime}-L^{\prime}$ is conserved by these intermediate scale interactions. We further assume that there are additional effects in the theory that link the asymmetries in the dark and visible sector, so that the true symmetry of the theory is $B-L-\left(B^{\prime}-L^{\prime}\right)$. Since it is expected that all global symmetries are violated by

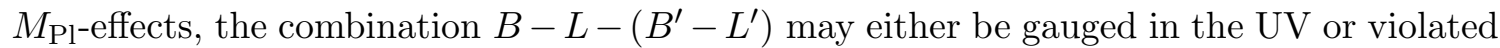
only by $M_{\mathrm{Pl}}$ effects that are not important for phenomenology.

Various potential genesis mechanisms have been outlined in the literature, which could be employed to generate the particle asymmetries e.g. [33-35]. We shall suppose that the asymmetries in $B^{\prime}$ and $L^{\prime}$ are of comparable magnitude, and that there is no overall asymmetry in the quantity $B-L-\left(B^{\prime}-L^{\prime}\right)$. For sufficiently high annihilation crosssections, the $B^{\prime}$ and $L^{\prime}$ states remain chemically coupled to the thermal bath long enough for Boltzmann suppression to remove most of their symmetric components. This results in $\overline{B^{\prime}}$ and $\overline{L^{\prime}}$ having highly suppressed relic abundances, whilst the yields of $B^{\prime}$ and $L^{\prime}$ are set by the particle-antiparticle asymmetries

$$
\frac{n_{B^{\prime}}}{n_{\gamma}} \simeq \eta_{B^{\prime}}, \quad \frac{n_{L^{\prime}}}{n_{\gamma}} \simeq \eta_{L^{\prime}} .
$$

We will also assume that $\eta_{B^{\prime}} \simeq \eta_{L^{\prime}}$, so that we end up with a relic population of $B^{\prime}$ and $L^{\prime}$ rather than their anti-particles. Asymmetries with different signs and magnitudes are also possible (and may be brought about dynamically, as discussed below), but we will not consider such models in this paper.

For such a scenario to lead to annihilation signals we propose that $B^{\prime}$-violating operators, suppressed by some intermediate scale, induce the decay of $B^{\prime}$ to $\overline{L^{\prime}}$, which subsequently annihilate with the population of $L^{\prime}$ resulting in observable signals. This scenario is illustrated schematically in figure 1 . This is inspired by proton decay due to GUT scale physics [36], from exchange of an $X, Y$ boson, inducing a decay $p \rightarrow e^{+}+\pi^{0}$ which is $B$ and $L$ violating but $B-L$ conserving. The proton lifetime is thus dependent on the GUT scale, the scale at which $B$ is perturbatively violated

$$
\tau_{p} \sim \frac{M_{\mathrm{GUT}}^{4}}{\alpha_{\mathrm{GUT}}^{2} m_{p}^{5}} .
$$




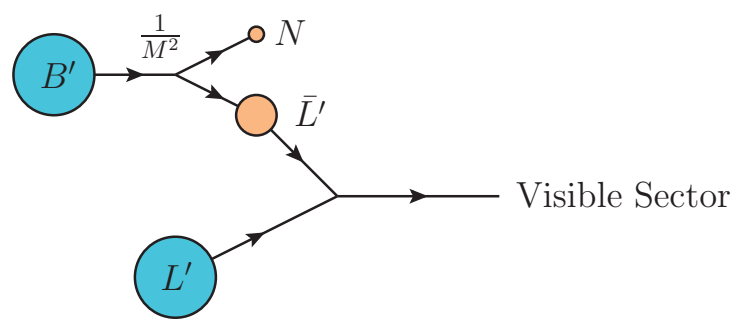

Figure 1. Schematic illustration of steps leading to annihilation signal. Initially, $B^{\prime}$ and $L^{\prime}$ are the only states in the hidden sector with non-negligible abundances. At some later time, such that it occurs in the current era, $B^{\prime}$ decays to $\overline{L^{\prime}}$ along with additional light states labelled $N$. The $\overline{L^{\prime}}$ subsequently (on galactic time scales) annihilate with $L^{\prime}$ to visible sector states leading to potentially observable signals.

We expect a similar expression for the lifetime of $B^{\prime}$ decaying to $\overline{L^{\prime}}$, dependent on the scale of $B^{\prime}$ violation (to some appropriate power, set by the dimension of the decay operator).

The case of particular interest is when the decays $B^{\prime} \rightarrow \overline{L^{\prime}}+\cdots$ (where the ellipsis denotes additional, relatively light, decay products) are slow such that this process occurs after the (dark) matter has coalesced into galaxies and clusters. If the decay products acquire too much kinetic energy, they will travel faster than galactic escape velocity. In this case, as discussed later, they are unlikely to annihilate on their way out of the galaxy, resulting in either decay-type profiles or no observable signals. The maximum kinetic energy available is determined by the mass splitting $\Delta m=m_{B^{\prime}}-m_{L^{\prime}}$, and a natural way to ensure that the final velocities are low enough, so to enable observable annihilation signals, is to have $\Delta m$ small relative to the DM mass. Such small mass splittings can arise for instance through radiative mass splitting between different DM 'flavours'. We discuss this constraint more generally in the next section, and outline two models which exhibit small mass splittings in section 4. Additionally, the small mass splitting will suppress the $B^{\prime}$ decay rate. For example, if the decay is via a dimension- 6 operator induced by $B^{\prime}$-violating physics at the scale $M_{\mathbb{B}^{\prime}}$, the lifetime will be (parametrically)

$$
\tau_{B^{\prime}} \sim 4 \pi \frac{M_{B^{\prime}}^{4}}{\alpha^{2}(\Delta m)^{5}},
$$

where $\alpha$ is the coupling associated to the $B^{\prime}$-violating physics.

The fact that the $B^{\prime}$ decay rate is suppressed (by powers of $\Delta m$ ) relative to symmetryviolating processes at higher energies means that it is necessary to check that the processes in the early universe do not alter the $B^{\prime}$ and $L^{\prime}$ asymmetries in undesirable ways. Indeed, as discussed in appendix $\mathrm{A}$, if these interactions are in equilibrium after the asymmetries have been established, they force $\eta_{B^{\prime}}=-\eta_{L^{\prime}}$, rather than the same-sign asymmetries required for the models discussed here. Consequently, the asymmetry must be set sufficiently late, such that these symmetry-violating processes are always out-of-equilibrium. Additionally, 
we assume that these asymmetries are set before freeze-out, ${ }^{2}$ leading to an upper limit on $\Gamma$ (equivalently, a lower limit on $\Delta m$ ). We shall examine these constraints shortly and demonstrate that viable models can be constructed.

\section{General constraints and signals}

Having introduced the general structure of the models considered, we now examine the relevant constraints and highlight particular features required for observable annihilation signals to arise in such models. For a given $B^{\prime} \rightarrow \overline{L^{\prime}}+\cdots$ decay rate $\Gamma$, the $\overline{L^{\prime}}$ population builds up (essentially) linearly over time until a significant fraction of the $B^{\prime}$ have decayed (unless the annihilation cross section is extremely high, only a small proportion of the $\overline{L^{\prime}}$ population that is regenerated ever annihilates). This $\overline{L^{\prime}}$ population translates into an upper bound on the $L^{\prime} \overline{L^{\prime}}$ annihilation cross section from astrophysical indirect detection signals (both late-time and recombination-time). A lower bound on the annihilation cross section comes from the requirement that the relic symmetric component is sufficiently depleted due to annihilations in the early universe. Further, demanding perturbative couplings places an additional upper bound on the cross sections.

These constraints fit together as illustrated in figure 2, which shows the allowed regions in terms of the (velocity independent) annihilation cross-section $\langle\sigma v\rangle \equiv \sigma_{0}$, and the $B^{\prime} \rightarrow$ $\overline{L^{\prime}}+\cdots$ decay rate $\Gamma$. The indirect detection constraints will depend on the DM mass (which sets the number density, and energy injection from annihilation), and on the annihilation channel (here, we show annihilation to muons - alternative channels will generally improve the CMB bounds by a factor of less than 3 , and the gamma-ray bounds by less than a factor 10). The perturbativity bound corresponds to a particular choice of model (see section 3.3), while the relic annihilation bound is weakly dependent on the DM mass. We observe that there is a significant amount of parameter space in which viable models can implemented. The potential signal region is the area close to the current gamma-ray limits, which can be probed by current and future experiments. Also, it can be seen that relatively slow decays are required to evade $\mathrm{CMB}$ limits. If $\Gamma t_{0} \gtrsim \frac{t_{0}}{t_{\mathrm{CMB}}} \sim 5 \times 10^{-5}$ (where $t_{0}$ is the current age of the universe, and $t_{\mathrm{CMB}}$ is the age at the recombination era), corresponding to the region at the far right of the diagram, then there is only a very small region of allowed parameter space. Current CMB observations are far enough above the cosmic variance limit [29-32] that future experiments should be able to close this gap for most decay channels.

A further possible constraint arises from DM self-scattering bounds (see e.g. [40-43]). The relationship between the annihilation cross section and the self-scattering cross-section is model-dependent, so may or may not introduce constraints within the perturbative region. In the model with a light hidden sector mediator considered in section 3.3, the scattering cross section can be sufficiently large to introduce limits. If we drop the assumption of perturbativity, then since the self-scattering cross section will generally be comparable to or larger than the annihilation cross-section, we obtain an upper bound on $\sigma_{0}$ of at most around 0.04 barn $\times \frac{m_{\mathrm{DM}}}{10 \mathrm{GeV}}$. This is low enough that, if the $\overline{L^{\prime}}$ is ejected from

\footnotetext{
${ }^{2}$ The alternative case of the asymmetry being set by decays after freeze-out is potentially interesting, however we will not examine such models of here.
} 


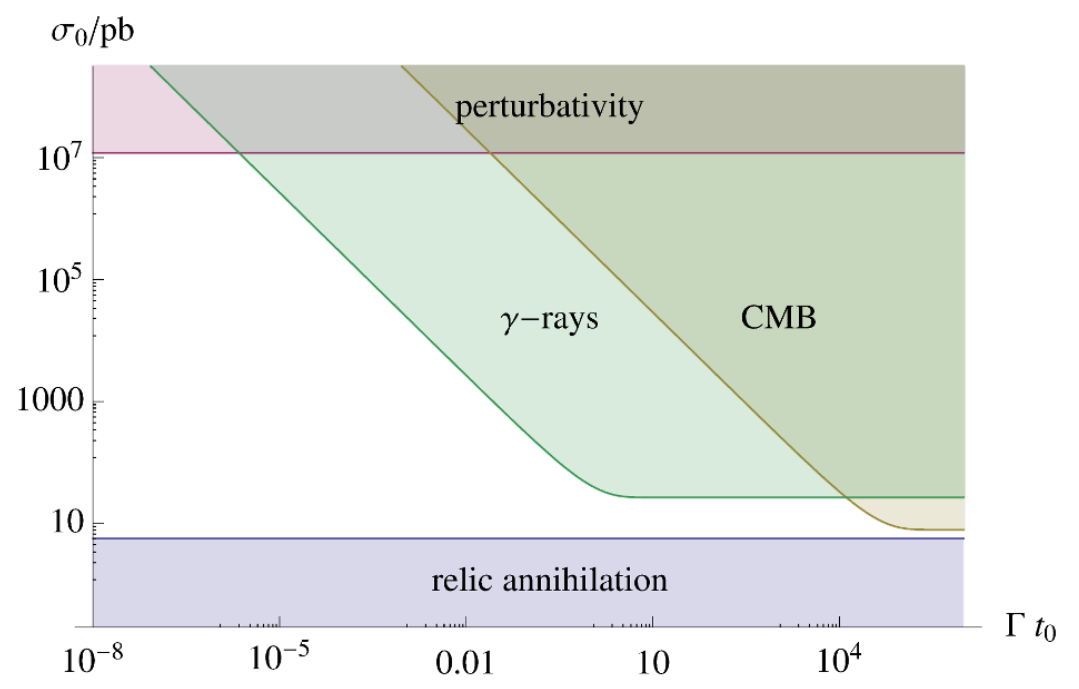

Figure 2. Constraints on $B^{\prime} \rightarrow \overline{L^{\prime}}+\cdots$ decay rate $\Gamma$ and $L^{\prime} \overline{L^{\prime}}$ annihilation cross section $\sigma_{0}$, with $m_{L^{\prime}}=10 \mathrm{GeV}$, assuming annihilation to muons. The perturbativity bound corresponds to the model of section 3.3, where $L^{\prime} \overline{L^{\prime}}$ annihilate into a pair of hidden sector vector particles, which then decay to the SM. Here, $t_{0}$ is the current age of the universe. The relic annihilation bound corresponds to the annihilation cross-section needed to annihilate all but 1 percent of the symmetric component, i.e. to obtain $r_{\infty} \equiv \frac{n_{\overline{B^{\prime}}}}{n_{B^{\prime}}}<0.01$. The CMB bound corresponds to the limit on energy injection from $L^{\prime} \overline{L^{\prime}}$ annihilation derived from CMB perturbations [29-32], while the gamma-ray bound is derived from FERMI observations [37] (assuming an NFW profile for the DM halo). We assume that the $\overline{L^{\prime}}$ injection velocity is small enough that the $\overline{L^{\prime}}$ distribution is similar to the $L^{\prime}$ one for the purposes of the gamma-ray constraints; we use the FERMI diffuse observations rather than the galactic centre observations, which would be less robust to profile modifications (see section 3.1).

the galaxy, only a small fraction of it will annihilate on the way out - consequently, the spatial distribution of annihilations will be approximately $\propto n_{L^{\prime}}$, so will resemble a decay profile. ${ }^{3}$ Thus, large annihilation cross sections do not remove the need for a small velocity kick if we want to generate an annihilation-type gamma-ray profile.

As we will discuss in section 3.2, some model-building is required to make sure that direct constraints (from collider and direct-detection experiments) do not place strong upper bounds on $\sigma_{0}$. For DM-SM interactions via contact operators, these bounds are generally (for $m_{\mathrm{DM}} \lesssim 30 \mathrm{GeV}$ ) below the lower bound from relic annihilation, so would leave no allowed parameter space. Figure 2 can be thought of as corresponding to the model of section 3.3, where $L^{\prime} \overline{L^{\prime}}$ annihilate to a pair of hidden sector particles, which then decay to the SM. In this case, direct constraints can be completely absent.

\section{1 $\quad B^{\prime}-L^{\prime}$ mass splitting and $\overline{L^{\prime}}$ distribution}

Unless the velocity of the decay-produced $\overline{L^{\prime}}$ is low enough, these states will be ejected from the galaxy, and not sufficiently concentrated to give detectable annihilation-type

\footnotetext{
${ }^{3}$ Such models in which annihilations produce decay-type gamma-ray profiles may be interesting in themselves, though we have not investigated them in any detail.
} 
signals. The escape velocity for the Milky Way is $\sim 500 \mathrm{~km} \mathrm{~s}^{-1}$, so the fractional $B^{\prime}-L^{\prime}$ mass splitting (assuming two-body decay, with the other product having much smaller mass) should satisfy

$$
\frac{\Delta m}{m_{L^{\prime}}} \lesssim \frac{500 \mathrm{~km} \mathrm{~s}^{-1}}{c} \approx 2 \times 10^{-3}
$$

For mass splittings much smaller than this, the $B^{\prime}$-to- $\overline{L^{\prime}}$ velocity change will be small compared to the velocity dispersion of the $B^{\prime}$, so the distribution of the $\overline{L^{\prime}}$ will be close to that of the $B^{\prime}$, and annihilation signals will have the standard $\propto n_{B^{\prime}}^{2}$ profile. However, for intermediate mass splittings, the extra velocity will be significant, and will result in an altered distribution for the $\overline{L^{\prime}}{ }^{4}$ Qualitatively, the distribution will be 'puffed out', with clumpiness being smoothed out and, moreover, systems with low escape velocities (e.g. dwarf galaxies) will not develop a bound population of $\overline{L^{\prime}}$ states.

Going beyond the two-body decay case, we will obtain a distribution of $\overline{L^{\prime}}$ velocity kicks, so some fraction of the $\overline{L^{\prime}}$ will be given higher-than-escape velocities. However, in general it is still the case that, as long as the other final or intermediate states do not have masses close to $\Delta m$, very few of the $\overline{L^{\prime}}$ end up with significantly smaller velocities than $\Delta m / m_{L^{\prime}}$. For example, if $\Delta m=0.1 m_{L^{\prime}}$, then the fraction of the $\overline{L^{\prime}}$ getting velocity kicks of less than $2 \times 10^{-3}$ is generally smaller than $5 \times 10^{-6}$ (see appendix B for details). So, in the absence of other 'coincidences' assuring a small velocity kick, a small mass splitting is needed to obtain significant $\overline{L^{\prime}}$ bound populations. As discussed in appendix B, instead of tuning the $B^{\prime}$ and $L^{\prime}$ masses to be close to each other, we could also tune the masses of the other decay products or intermediate states; however, a small mass splitting is more natural in many model-building contexts.

Quantitatively, figure 3 shows an approximation to the $\overline{L^{\prime}}$ profiles obtained for different injection velocities, starting from an NFW-type profile. As described in appendix C, we calculate these by convolving the initial DM phase-space distribution function with a velocity-kick kernel, then reparameterising to find the new steady-state distribution function, and integrating over this to find the new spatial number density. We see, as expected, that the deviation from the distribution of the parent particle is small for injection velocity smaller than the $B^{\prime}$ velocity dispersion, with the profile being flattened out for larger $v_{i}$. For the example in figure 3 the profiles of states produced with $v \lesssim 50 \mathrm{~km} \mathrm{~s}^{-1}$ remain approximately NFW and for increasing injection energies the profiles are smooth deformations away from the profile of the parent particle. In section 3.5, we study the effect of this on the observed annihilation profile and the compatibility with tentative signals that may have recently been observed.

\footnotetext{
${ }^{4}$ As relaxation times for galaxies such as the Milky Way are large enough that stellar encounters are generally unimportant [45], the extra energy will not be removed by gravitational interactions. Also, the bounds on DM self-scattering [40-43] mean that a given particle can interact at most a few times over galactic timescales, so it is only at the upper end of the allowed scattering cross section region that appreciable energy is lost this way. Such large self-scattering cross-sections also have consequences for the dominant component of the DM distribution (pushing the halo towards isothermality), and we will not consider the details of such models here.
} 


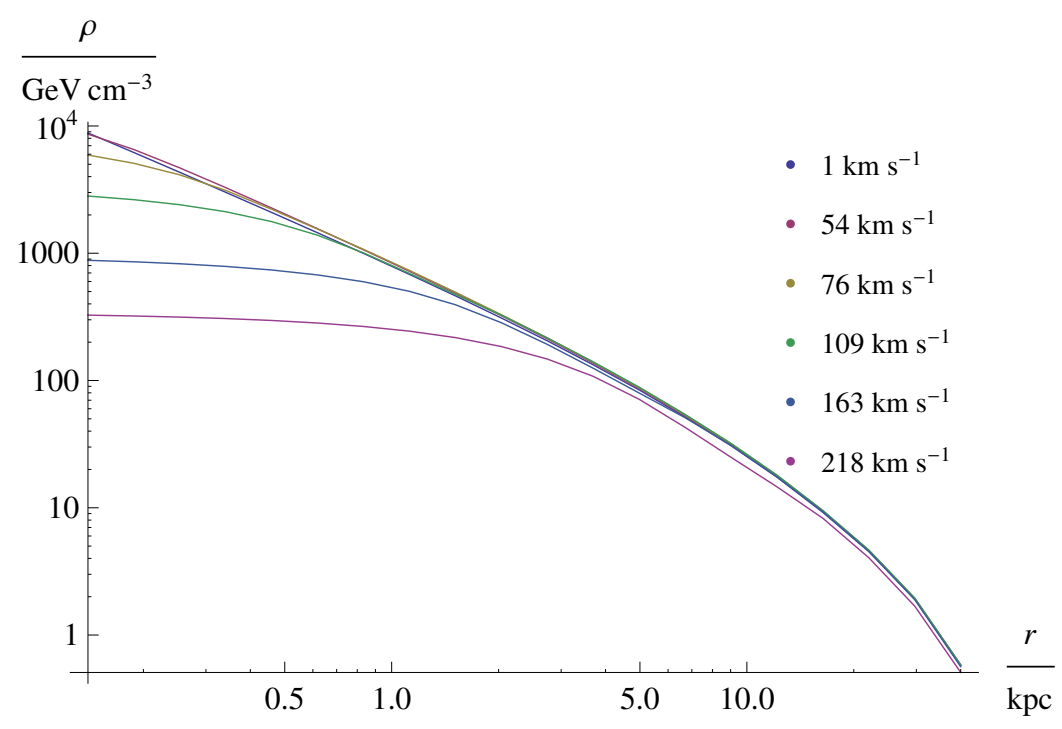

Figure 3. Approximation to $\overline{L^{\prime}}$ profile after injection at relative velocity $v_{i}$ as listed, starting from a NFW-type $B^{\prime}$ distribution with shape parameter $\gamma=1.2\left(\right.$ so $\rho \propto r^{-1.2}\left(1+r / r_{s}\right)^{-1.8}$, taking $r_{s}=20 \mathrm{kpc}$ ). The $B^{\prime}$ distribution is taken to have isotropic velocity distribution, which implies velocity dispersion of $\sigma=94 \mathrm{~km} \mathrm{~s}^{-1}$ at $r=1 \mathrm{kpc}$. As described in appendix C, we approximate the $\overline{L^{\prime}}$ distribution as also having isotropic velocity distribution, which will make the larger- $v_{i}$ profiles slightly less peaked than they should be.

There is also the possibility of indirect signals from the $B^{\prime} \rightarrow \overline{L^{\prime}}+\cdots$ decays themselves, if the other decay products include SM states [47]. In figure 4 we show conservative ('worst-case') constraints on the decay rate in this scenario; we assume that all decays proceed via $B^{\prime} \rightarrow \overline{L^{\prime}}+\gamma \gamma$, producing a sharp spectrum of photons and maximising the detectability of the signal. Note that, since the bounds from decays to electrons are at most a factor of $\sim 100$ worse than from those to photons [47], then in the absence of a small $(\lesssim 0.1 \mathrm{MeV})$ mass splitting, if annihilation signals are to dominate over decays it is a requirement that most of the energy from decays is dumped into other hidden sector states (or into neutrinos). Otherwise, since only a small proportion of the emitted $\overline{L^{\prime}}$ have annihilated by the present day, the SM states emitted in every decay will be a stronger signal. This can impose an extra constraint on our model building from the requirement of having additional light stable hidden sector states, and ensuring these have sufficiently small relic abundance. Such light states can arise through small Dirac or Majorana masses, or as pseudo-goldstone bosons.

If the $B^{\prime}$ lifetime is not much longer than the age of the universe, then sufficiently large velocity kicks from its decay can affect the structure formation process. The strongest constraints on small velocity kicks $\left(v_{i} \lesssim 100 \mathrm{~km} \mathrm{~s}^{-1}\right)$ comes from their effect on the population of Milky Way satellite galaxies [48, 49]. Such constraints depend on modelling of the highly non-linear regime of structure formation, but constraints from Lyman- $\alpha$ observations (which probe much earlier times) are almost as limiting [50]. Figure 4 shows the galactic bounds in the $(\Delta m, \Gamma)$ plane (assuming $v_{k} \approx \Delta m / m_{B^{\prime}}$ ). 


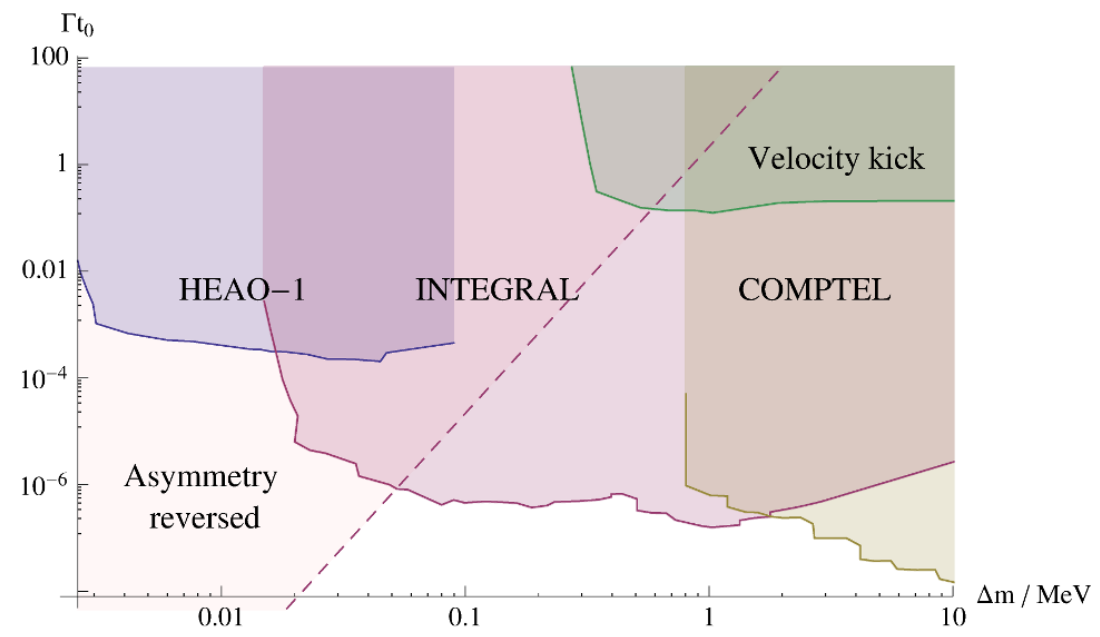

Figure 4. Constraints on the $B^{\prime} \rightarrow \overline{L^{\prime}}+\cdots$ decay rate $\Gamma$ (compared to the age of the universe $t_{0}$ ) from X-ray observations, for $m_{B^{\prime}}=10 \mathrm{GeV}$, assuming that all decays are $B^{\prime} \rightarrow \overline{L^{\prime}}+\gamma \gamma$ and that the DM profile is NFW; constraints from the HEAO-1 (blue), INTEGRAL (red), and COMPTEL (yellow) experiments [46] are shown. The green region corresponds to velocity kicks (assuming $\left.v_{i} \approx \Delta m / m_{B^{\prime}}\right)$ ruled out by structure formation constraints [48, 49]. The region above the dotted line is not viable in the model of section 4.1 , since $B^{\prime}$ and $L^{\prime}$ violating interactions stay in equilibrium until $T \lesssim 50 \mathrm{GeV}$ (see appendix A).

As commented on in the previous section, the requirement that the asymmetries in $B^{\prime}$ and $L^{\prime}$ are not disrupted through symmetry-violating processes in the early universe places a lower bound on $\Delta m$ (equivalently, upper bound on $\Gamma$ ). In figure 4 we also show the approximate bound from requiring that symmetry-violating interactions decouple sufficiently early, as discussed in appendix A. The mass splittings in figure 4 are related to the velocity kicks, e.g. as studied in figure 3 by eq. (3.1). For example, taking $m_{B^{\prime}}=10 \mathrm{GeV}$, the value used in the figure, $\Delta m \approx 2 \mathrm{MeV}(7.2 \mathrm{MeV})$ corresponds to a velocity kick in the region of $v=54 \mathrm{~km} \mathrm{~s}^{-1}\left(218 \mathrm{~km} \mathrm{~s}^{-1}\right)$. We can see that there is a significant allowed region for all cosmologically-slow decay rates and mass splittings in the range of interest. However, in combination with the velocity kick bounds described in the previous paragraph, cosmologically early decays are generally excluded, independently from the previously mentioned direct detection bounds.

\subsection{Direct and indirect detection}

In addition to the gamma ray constraints arising from late-time annihilations, there is also the prospect of signals coming from annihilation products in cosmic rays (for the low DM mass range we consider, annihilations into neutrinos will generally be beyond the reach of near-term experiments ${ }^{5}$ ). DM annihilation into quarks results in cosmic ray antiprotons,

\footnotetext{
${ }^{5}$ If the DM is sufficiently strongly self-interacting, and has some scattering cross-section with nuclei, there is the possibility of building up a large population within the Sun, annihilations within which may be detectable by neutrino observatories $[54,55]$. Up to some threshold, lower annihilation rates will actually increase this signal (by allowing a larger equilibrium DM population in the Sun), so such signals could arise for decay rates much slower than the age of the universe.
} 


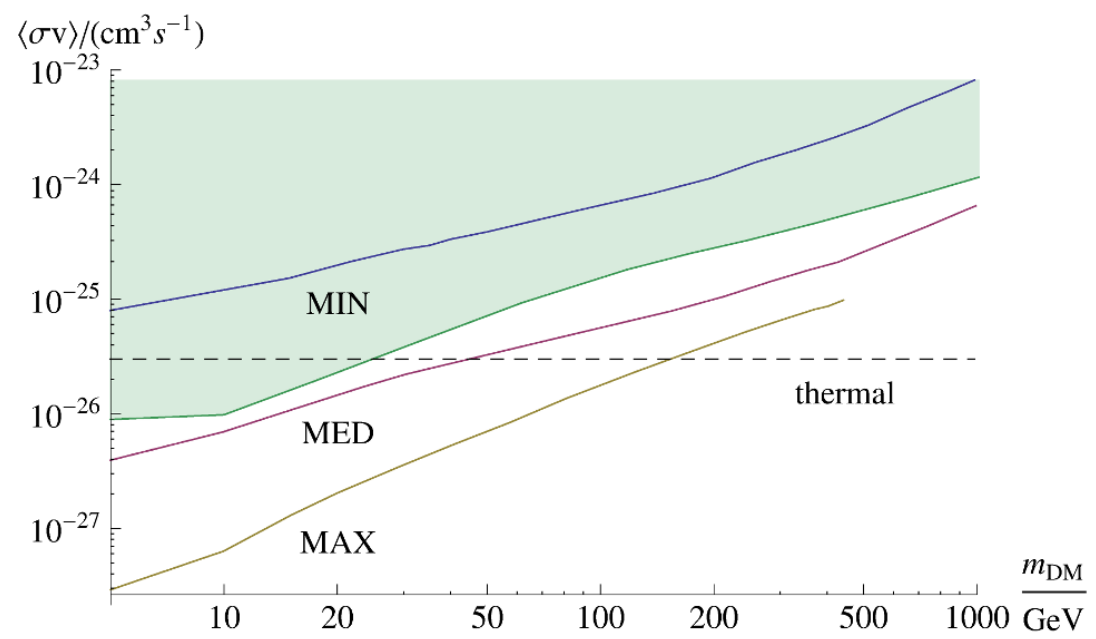

Figure 5. Limits on symmetric DM annihilation cross section to $b \bar{b}$ from (blue, red, yellow) PAMELA cosmic ray antiproton measurements [51], under different assumptions with respect to propagation of charged particles through the galaxy, and (green) FERMI gamma-ray observations [37]. (Note that the PAMELA bounds correspond to assuming that the DM halo has an Einasto density profile, while the FERMI bounds assume a NFW profile; however, the difference will be minor [52].)

the population of which has been measured by the PAMELA experiment (and will also be measured by AMS-02) [51]. Since antiprotons are charged particles, whose trajectories are affected by galactic magnetic fields, their direction of arrival is not simply related to the location of their source, and very little can be inferred about the galactic DM distribution from such measurements. In addition, there is a considerable degree of uncertainty as to exactly how this propagation through the galaxy occurs, and different models result in significantly different derived constraints on DM annihilation rates [52]. Figure 5 illustrates how the antiproton limits compare to those from gamma-ray observations, under three different propagation scenarios (MIN/MED/MAX) that are standard in the literature, see e.g. [51]. The limits range from significantly less constraining than those from gammarays, to very significantly more so. Consequently, depending on the true propagation, it is possible that annihilation signals may first be observed in either antiprotons or gamma signals.

Similar considerations apply to cosmic ray positrons, which are produced by annihilation into leptons (and also by annihilation to EW gauge bosons). For annihilation into electrons or muons, the constraints on the DM annihilation cross section from positron observations (for $m_{\mathrm{DM}} \lesssim 100 \mathrm{GeV}$ ) are generally significantly stronger than those from gamma-ray observations (see e.g. figure 3 of [53]) and CMB perturbations. For annihilation to taus, the constraints are comparable.

In addition to the limits from indirect detection, models of ADM are constrained by direct detection experiments and collider searches for events with missing energy. Specifically, in order for a particle asymmetry to set the relic density, and thus to obtain ADM, the symmetric component must annihilate efficiently (with a cross section larger than that 
required for symmetric freeze-out) and since these couplings also set the production and scattering cross sections this can lead to tension with experimental searches. In particular if the annihilation of the symmetric component is directly to SM states and can be described via contact operators (i.e. from integrating-out some heavy mediator) then this generally results in fairly strong constraints:

- Annihilation via contact operators involving SM quarks was studied in [22] (see also [21]). It was argued that direct detection experiments and LHC monojet limits typically exclude models of ADM for $m_{\mathrm{DM}} \lesssim 100 \mathrm{GeV}$.

- LEP searches for mono-photon events [20] constrain couplings between DM and electrons. For universal couplings of DM to charged leptons they exclude the ADM parameter region for $m_{\mathrm{DM}} \lesssim 30 \mathrm{GeV}$. Preferential annihilation to $\mu$ or $\tau$ leptons is not constrained.

- [27] considered the limits from collider observations of DM interactions with electroweak gauge bosons, excluding ADM models for $m_{\mathrm{DM}} \lesssim 40 \mathrm{GeV}$.

These collider limits can be circumvented if the model features a 'light' mediator state [1$3,22]$ (relative to collider energies i.e. $\lesssim 100 \mathrm{GeV}$ for LHC searches). In the remainder of section 3, we study two illustrative examples in which we can build perturbative models that significantly alleviate these constraints:

- Annihilation of the symmetric component to (on-shell) metastable vector bosons ${ }^{6}$ $L^{\prime} \overline{L^{\prime}} \rightarrow V V$, with $V$ subsequently decaying to SM states (similar to [56]).

- Annihilation via the s-channel process $L^{\prime} \overline{L^{\prime}} \rightarrow \phi \rightarrow$ SM involving a pseudoscalar mediator $\phi$, with $m_{\phi} \approx 2 m_{L^{\prime}}$ such that the cross section is resonantly enhanced, cf. [22].

\subsection{Annihilation via $L^{\prime} \overline{L^{\prime}} \rightarrow V V$}

Let us consider the case that $L^{\prime}$ is a fermion and there is a the hidden sector vector boson $V$ which acts as a mediator state. For $m_{V}<m_{L^{\prime}}$, annihilation to a pair of on-shell vectors is possible $L^{\prime} \overline{L^{\prime}} \rightarrow V V$, and if $V$ has a small coupling to the SM, this channel will dominate. As an explicit example of this scenario, we consider the following interaction

$$
\mathcal{L} \supset \lambda\left(\overline{L^{\prime}} \gamma^{\mu} L^{\prime} V_{\mu}+\overline{B^{\prime}} \gamma^{\mu} B^{\prime} V_{\mu}\right)
$$

with $V$ subsequently decaying to light SM states. In figure 6 we illustrate how the constraints on $\lambda$ vary with $m_{V}$, for $m_{L^{\prime}} \simeq 10 \mathrm{GeV}$ and assuming that $V$ decays dominantly to muon pairs (such a coupling can arise due to kinetic mixing as we discuss in section 4). We assume here that the $V$ lifetime is short enough that is does not travel an astronomically

\footnotetext{
${ }^{6}$ The simple alternative with a scalar mediator $\phi$ (and $L^{\prime}$ a fermion) is not viable, as in this case the annihilation channel $L^{\prime} \overline{L^{\prime}} \rightarrow \phi \phi$ is suppressed by $v^{2}$. Whilst the symmetric component can be regenerated through early decays without conflict with CMB observables, as the DM velocity in the galaxy is $\sim 10^{-3}$, observably high galactic annihilation rates cannot be obtained for perturbative couplings in this model.
} 


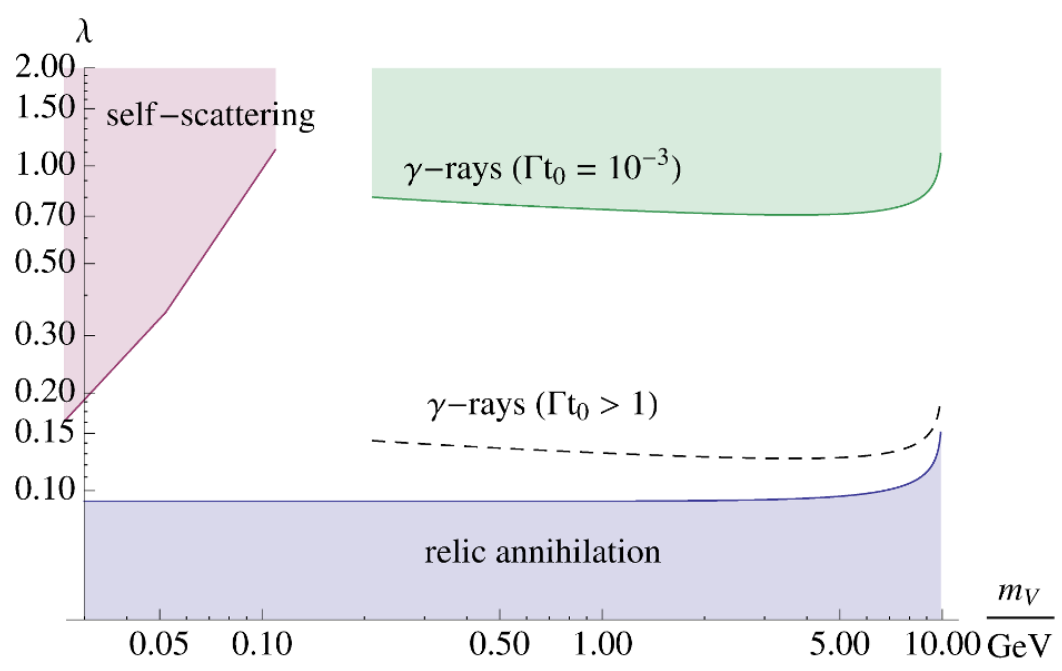

Figure 6. Constraints on $\lambda$ for $m_{L^{\prime}}=10 \mathrm{GeV}$, with $V$ decaying to $\mu^{+} \mu^{-}$. The red curve corresponds to the lower limit on $m_{V}$ from DM self-scattering [40], the blue curve shows the lower limit on $\lambda$ required for $r_{\infty}<0.01$ (i.e. for efficient annihilation of the ADM symmetric component), the dashed black curve shows the upper bound on $\lambda$ from FERMI gamma-ray bounds assuming that all of the $B^{\prime}$ have decayed by the present time $\left(\Gamma t_{0}>1\right)$, and the green region shows the gamma-ray bounds for the case where only a fraction $\Gamma t_{0}=10^{-3}$ of the $B^{\prime}$ have decayed. See figure 2 for discussion of the gamma-ray bounds. Decays of $V$ to $e^{+} e^{-}$would result in broadly similar gamma-ray constraints, and would allow $m_{V}$ down to the self-scattering bound (note that, close to the self-scattering bound, the gamma-ray limits would be altered, since the assumption of a NFW halo is not self-consistent here [40-43]).

significant distance before decaying - if it is longer lived, then the drift of the $V$ from the annihilation point can modify the observed profile, as discussed in [38] (though in that case, we would need to worry about its relic abundance and decays in the early universe). The cascade structure of the annihilation means that the resulting gamma-ray spectrum is softer than that arising from direct annihilations to muons [39] (as taken into account approximately in figure 6), but since the low-energy part of the spectrum is important in setting bounds on the annihilation rate at DM masses this low [37], this does not affect the constraints very significantly. Note that $V$ exchange contributes to DM-DM scattering and, since the self-scattering cross section increases with decreasing $m_{V}$, limits on the DM self-interaction [40] (see also [41-43]) give a lower bound on $m_{V}$, as indicated in figure 6 . Further constraints on this example are encapsulated in figure 2; for the models shown the decays are cosmologically slow in order to evade CMB bounds. Notably, for a wide range of decay rates and mediator masses, models consistent with experimental constraints exist.

If $m_{V}$ is sufficiently small, then the long-range $V$-exchange between $L^{\prime}$ and $\overline{L^{\prime}}$ can give rise to Sommerfeld-type low-velocity enhancement of the annihilation rate. However, the lower limit on $m_{V}$ from self-scattering implies that this is not significant here. Also, as discussed in section 3.1, DM self-scattering cross sections near to the observational limit (as sometimes invoked to solve problems with small-scale structure formation [57]) have consequences for the shape of the DM halo, though we do not consider the details here. 


\subsection{Annihilation via $L^{\prime} \overline{L^{\prime}} \rightarrow \phi \rightarrow \mathrm{SM}$}

We now turn to the second model, in which $L^{\prime} \overline{L^{\prime}}$ annihilation proceeds through the schannel exchange of a mediator $\phi$, where $m_{\phi}$ is close to resonance. For the case where $m_{\phi}>m_{L^{\prime}}$, the $L^{\prime} \overline{L^{\prime}}$ annihilations cannot produce on-shell $\phi$ pairs and rather proceed directly to SM states via a $\phi$-mediator. Since we expect $L^{\prime}$ to be roughly $\mathrm{GeV}$ or greater, the DM-proton scattering cross section is well described by an effective operator and if $\phi$ is a scalar or a vector, coupling to quarks, then large regions of parameter space are excluded by direct detection experiments, see $[22]{ }^{7}$ Whilst the effective theory is valid for DM-proton scattering, kinematic effects can be important to the annihilation process and can potentially lead to regions of parameter space which are not excluded by direct searches, as we discuss below.

Given $\phi$-DM and $\phi$-SM couplings, the $L^{\prime} \overline{L^{\prime}} \rightarrow$ SM annihilation cross section is dramatically increased if $m_{\phi} \approx 2 m_{L^{\prime}}$, such that the s-channel annihilation is close to resonance. This does not make the direct search constraints tighter, so gives us more parameter space for the model. In particular, if $m_{\phi}$ is slightly above $2 m_{L^{\prime}}$, then at higher temperatures the thermal averaging samples more of the resonance peak, increasing the annihilation cross section. Effectively an enhancement of $\langle\sigma v\rangle$ at high temperatures occurs, as required for the early-decay case to be viable.

To illustrate the allowed parameter space around the resonance region, we consider an explicit model with a pseudoscalar $\phi$ coupling to quarks via

$$
\mathcal{L} \supset i \lambda \phi \overline{L^{\prime}} \gamma^{5} L^{\prime}+\sum_{q} i \lambda^{\prime} y_{q} \frac{m_{\phi}}{m_{h}} \phi \bar{q} \gamma^{5} q
$$

Figure 7 shows the constraints in the $\left(m_{\phi}, \lambda\right)$ plane for $\lambda^{\prime}=0.14$ (the behaviour is mostly dependent on $\lambda \lambda^{\prime}$, so we only plot variation with $\lambda$ ). As discussed above, there is a strong distinction between $m_{\phi}$ just above vs below $2 m_{L^{\prime}}$. Above, the enhancement of the annihilation cross section with temperature means that there is a large allowed region between the couplings necessary for relic annihilation and those ruled out by astrophysical constraints. For $m_{\phi}<2 m_{L^{\prime}}$, on the other hand, higher collision energies result in $\phi$ being more off-shell. Consequently, in this region, the relic annihilation constraint rules out everything until $\lambda$ is very large.

The figure shows the strongest bounds, when the decays are early enough to maximise the limits from both CMB and galactic timescales. Since this still leads to viable parameter space, early decays are permitted (unlike the previous models, which as seen in figure 2 are very constrained if there are early decays). Note that, as we increase the decay time past recombination time, the CMB limits become less constraining relative to the gammaray bounds (cf. figure 2), so (depending on assumptions about antiproton propagation, cf. figure 5) there is a large parameter space in which gamma-ray signals may realistically be seen before other signals.

\footnotetext{
${ }^{7}$ More complicated portal interactions can alleviate some direct detection constraints, e.g. [23-26].
} 


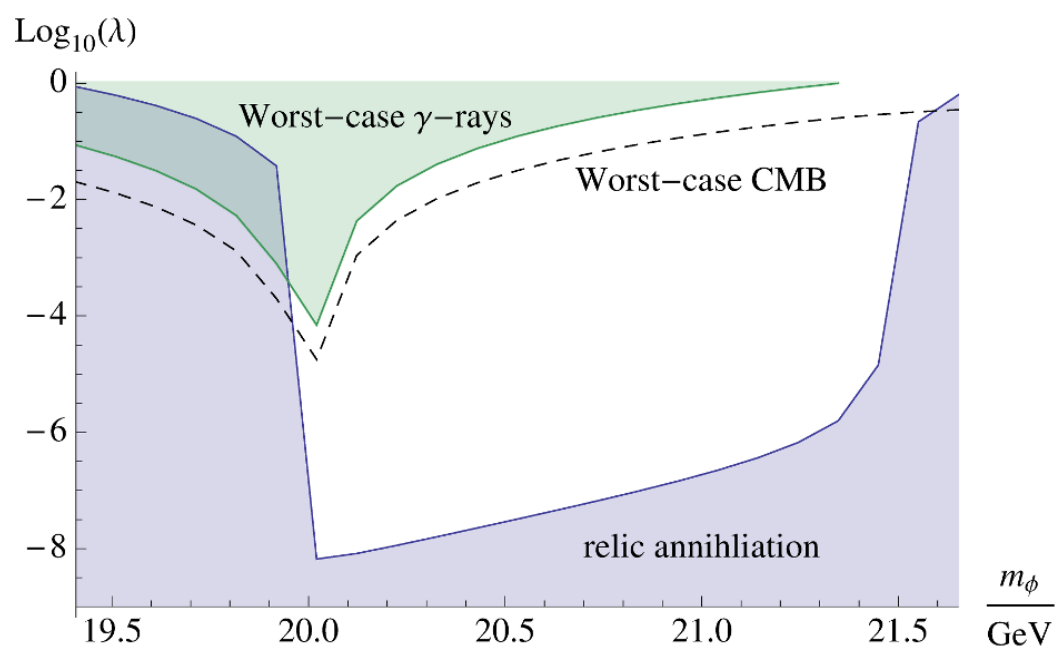

Figure 7. Constraints on $\lambda$ for $m_{L^{\prime}}=10 \mathrm{GeV}$, for coupling to quarks as described in section 3.4. The blue curve shows the lower limit on $\lambda$ required for $r_{\infty}<0.05$ (i.e. for efficient annihilation of the $\mathrm{ADM}$ symmetric component), and the green region is excluded by gamma-ray bounds (see figure 2 for details), assuming that all of the $B^{\prime}$ have decayed by the present time. The black dotted curve shows the exclusion bound from CMB perturbations, assuming that all of the $B^{\prime}$ have decayed by recombination time. The relic abundance and annihilation cross-section calculations were carried out using the micrOMEGAs package [58].

\subsection{Tentative signals and morphology}

Recently, there have been suggestions [59,60] (see also [61-65]) of a gamma-ray excess from the galactic centre and its vicinity, compatible with $\sim 10 \mathrm{GeV}$ DM (NFW-profile) annihilating to leptons (with $\langle\sigma v\rangle \simeq 2 \times 10^{-27} \mathrm{~cm}^{3} \mathrm{~s}^{-1}$ ) or with $\sim 50 \mathrm{GeV}$ DM annihilating to quarks (with $\langle\sigma v\rangle \simeq 8 \times 10^{-27} \mathrm{~cm}^{3} \mathrm{~s}^{-1}$ ). Both models discussed above can reproduce a signal of this kind, but only with a sufficiently small $B^{\prime}-L^{\prime}$ mass splitting such that the $\overline{L^{\prime}}$ profile remains sharply peaked towards the galactic centre. It was claimed in [59, 60] that, for symmetric annihilating DM, the signal is fit well by a generalised NFW type profile with shape parameter $\gamma \simeq 1.2$, and with some systematic uncertainty possibly allowing $\gamma$ to vary around this value to a maximum of $\sim 2$ (see also [66]).

Figure 8 shows the profile shapes resulting from different $B^{\prime} \rightarrow \overline{L^{\prime}}+\cdots$ injection velocities, starting from a NFW-type $(\gamma=1.2) B^{\prime}$ profile. These are calculated as described in appendix $\mathrm{C}$, utilising the properties of the steady-state DM phase space distribution function. As it illustrates, we need $v_{i} \lesssim 120 \mathrm{~km} \mathrm{~s}^{-1}$ to match a symmetric annihilation signal with $1.1<\gamma<1.3$, corresponding to a fractional $B^{\prime}-L^{\prime}$ mass splitting of $\lesssim 4 \times 10^{-4}$ (subject to the caveats mentioned in section 3.1 and appendix B). If the mass splitting is not much smaller than this limit, then the DM gamma-ray signals from structures with lower velocity dispersions than the galaxy will be modified compared to what we expect from standard annihilating symmetric DM. At present, the nature of these structures (e.g. DM clumps in the galactic halo) is poorly constrained, so it is hard to pick out any definite differences, but since the escape velocity for most dwarf galaxies is below $\sim 50 \mathrm{~km} \mathrm{~s}^{-1}$ [67], and the velocity dispersion is $\sim 10 \mathrm{~km} \mathrm{~s}^{-1}$ [68], if $v_{i}$ is high enough 

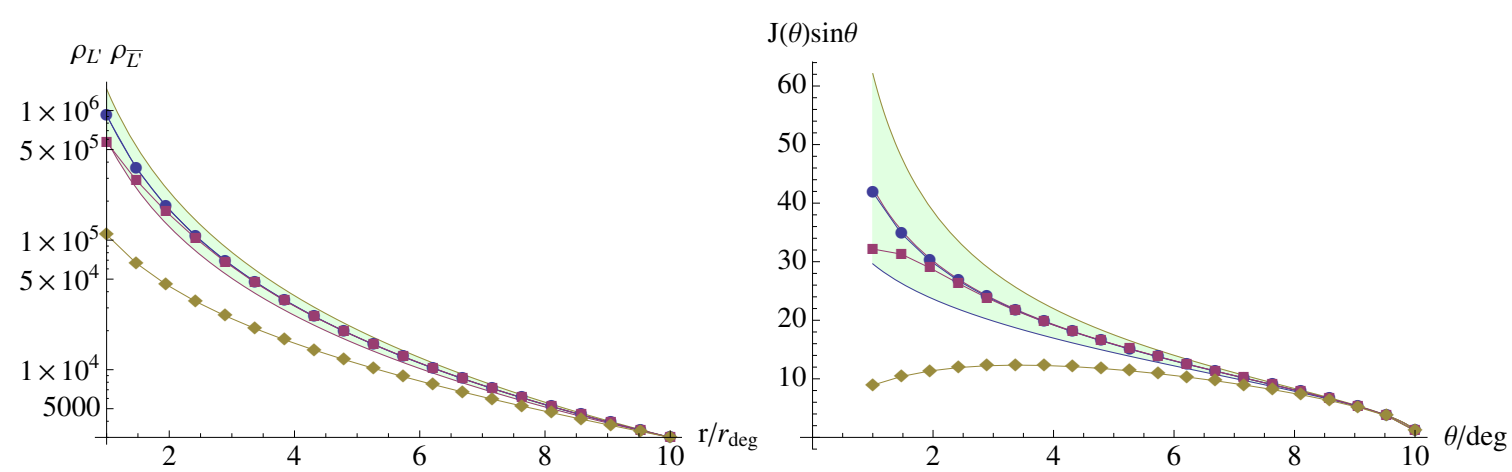

Figure 8. Left: the $\rho_{L^{\prime}} \rho_{\overline{L^{\prime}}}$ profile for different $L^{\prime}$ and $\overline{L^{\prime}}$ profiles (in arbitrary units, normalised to a common value at $r=10 r_{\mathrm{deg}}$, where a distance of $r_{\mathrm{deg}}$ above the galactic centre corresponds to $b=1^{\circ}$ ). The blue dots indicate the $\gamma=1.2 L^{\prime}$ profile, and a $\overline{L^{\prime}}$ profile obtained by starting with a NFW $\gamma=1.2$ profile and applying a velocity kick of $1 \mathrm{~km} \mathrm{~s}^{-1}$. The red and yellow dots correspond to the same situation, but with velocity kicks of $76 \mathrm{~km} \mathrm{~s}^{-1}$ and $163 \mathrm{~km} \mathrm{~s}^{-1}$ respectively. The shaded region corresponds to generalised NFW profiles (for $L^{\prime}$ and $\overline{L^{\prime}}$ ) with $1.1<\gamma<1.3$ required to best match the apparent gamma-ray excesses near the galactic centre [59,60]. Right: for a given model the signal size is determined by the integral $J(\theta)=\int d l \rho_{L} \rho_{\bar{L}}$ along the line-of-sight as a function of angle from galactic centre. Colours and shading as in left panel.

then we will almost completely suppress the annihilation signal from dwarf galaxies due to most of the $\overline{L^{\prime}}$ population escaping. However, with present and near-future experiments, gamma-ray observations of dwarf galaxies place significantly weaker constraints on DM annihilation cross sections than galactic centre observations [69], without the assumption of large boost factors due to DM clustering or velocity suppression. As a consequence, for annihilation cross sections at the sub-thermal level required to replicate the signal under discussion, we would not expect to see the corresponding signals from dwarf galaxies in the near future.

In addition to the modified $\overline{L^{\prime}}$ distribution in space, the main possibly-observable difference from a standard annihilating symmetric DM scenario is the change in $\overline{L^{\prime}}$ population over time. However, due to the small (symmetric) annihilation cross section required to match the signal, the effect on the CMB from symmetric annihilations would be below cosmic variance [29-32], so it would not be possible to detect the difference between this and a smaller effect in the $\overline{L^{\prime}}$ case. This is not a completely general statement - there do exist viable symmetric DM models which have observable annihilation effects on the CMB, and in these cases the late-decay ADM model would make different predictions. Also note that possible collider signals of an ADM model may differ from those of a symmetric DM model (though in neither case are we guaranteed to have such signals, e.g. the models of section 3.3 in our case, and the symmetric model of [70]).

\section{Model building}

It was argued in the previous section that a small mass splitting is a natural way to obtain observable annihilation signals. Hence, next we highlight a motivated setting in which such 


\begin{tabular}{|c|c||c||c|c|c|}
\hline & spin & $Z_{2}$ & $B^{\prime}$ & $L^{\prime}$ & $B^{\prime}-L^{\prime}$ \\
\hline$\phi$ & 0 & 1 & - & - & 0 \\
\hline$B^{\prime}{ }_{L \alpha}$ & $\frac{1}{2}$ & 1 & $1 / 2$ & 0 & $1 / 2$ \\
\hline$B_{R \alpha}^{\prime \dagger}$ & $\frac{1}{2}$ & 1 & $-1 / 2$ & 0 & $-1 / 2$ \\
\hline$L^{\prime}{ }_{L \alpha}$ & $\frac{1}{2}$ & 0 & 0 & $1 / 2$ & $-1 / 2$ \\
\hline$L_{R \alpha}^{\prime \dagger}$ & $\frac{1}{2}$ & 0 & 0 & $-1 / 2$ & $1 / 2$ \\
\hline$N_{1 \alpha}$ & $\frac{1}{2}$ & 1 & 0 & 0 & 0 \\
\hline$N_{2 \alpha}$ & $\frac{1}{2}$ & 0 & 0 & 0 & 0 \\
\hline
\end{tabular}

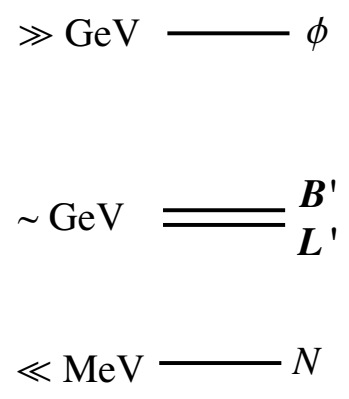

Table 1. Left: hidden sector fundamental matter content. Right: hidden sector mass hierarchy.

a scenario may occur. Specifically, we outline a model based on broken flavour symmetries in the hidden sector. Further, drawing on analogies with nuclear (proton-neutron) mass splitting, we sketch an alternative realisation of such a set-up in models of composite DM.

\subsection{An implementation with fundamental matter}

We first present a simple implementation involving (fundamental) matter that obtains Dirac masses, with a small mass splitting generated from radiative breaking of a flavour symmetry. The hidden sector has a $Z_{2}$ symmetry, an exact $B-L-\left(B^{\prime}-L^{\prime}\right)$ symmetry, and accidental approximate $B^{\prime}$ and $L^{\prime}$ symmetries. ${ }^{8}$ The matter content of our (example) model is displayed in table 1; it features a heavy complex scalar $\phi$ and fermion matter, which is written as two component left-handed Weyl spinors. The typical mass hierarchy of the states is displayed to the right of table 1 .

The Lagrangian involving the light fields can be expressed as

$$
\mathcal{L} \supset-m_{B^{\prime}} B_{L \alpha}^{\prime} B_{R}^{\prime \alpha}-m_{L^{\prime}} L_{L \alpha}^{\prime} L_{R}^{\prime \alpha}+\text { h.c. }
$$

Note that Majorana mass terms for $L^{\prime}, B^{\prime}$ are forbidden by the exact $B-L-\left(B^{\prime}-L^{\prime}\right)$ symmetry and mixing between these states is forbidden by the $Z_{2}$ symmetry. It can be seen that $B^{\prime}$ and $L^{\prime}$ arise as approximate symmetries since there are no renormalisable terms in the low energy effective theory that violate them. However, the heavy scalar $\phi$ has couplings of the form

$$
\mathcal{L} \supset y_{1} \phi\left(B_{L}^{\alpha} L_{L \alpha}+B_{R \dot{\alpha}} L_{R}^{\dot{\alpha}}\right)+y_{2} \phi N_{1}^{\alpha} N_{2 \alpha}+\text { h.c. }
$$

As a result, this can mediate decays of $B^{\prime}$ to $\overline{L^{\prime}}$ as shown in figure 9 . We shall assume that the scalar $\phi$ gains a mass well above $B^{\prime}$ and $L^{\prime}$ and hence can be integrated out, leading to a four-fermion operator. The lifetime of $B^{\prime}$ is given in eq. (2.3), setting $M_{B^{\prime}}=m_{\phi}$ and hence, for a given model, one can compare with the limits indicated in figure 2 .

\footnotetext{
${ }^{8}$ The couplings studied here actually respect $B^{\prime}-L^{\prime}$, however once a mechanism to cogenerate or share asymmetries with the visible and hidden sectors is included, the true symmetry is $B-L-\left(B^{\prime}-L^{\prime}\right)$.
} 


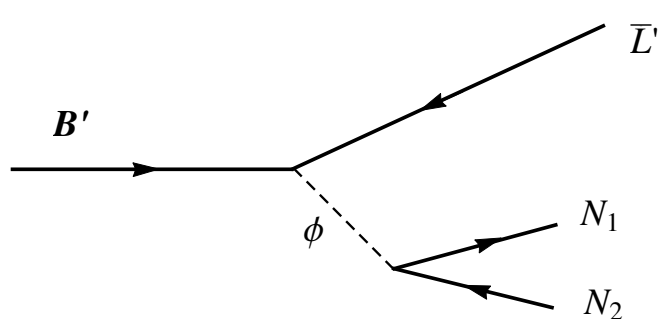

Figure 9. $\overline{L^{\prime}}$ are generated via $B^{\prime}$ decays and then annihilate with the asymmetric population of $L^{\prime}$.

The fields $N_{1}$ and $N_{2}$ are assumed to gain Majorana masses $M_{1}$ and $M_{2}$, which are parametrically smaller than the $B^{\prime}$ and $L^{\prime}$ (Dirac) mass scale. ${ }^{9}$

$$
\mathcal{L} \supset-M_{1} N_{1 \alpha} N_{1}^{\alpha}-M_{2} N_{2 \alpha} N_{2}^{\alpha}+\text { h.c. }
$$

Further, we assume that $m_{B^{\prime}}=m_{L^{\prime}}$ at leading order, exhibiting an approximate 'flavour' symmetry which is only radiatively broken. This breaking could be due to the differing $Z_{2}$ parities of these states, resulting in the $B^{\prime}$ and $L^{\prime}$ having different couplings to some additional (possibly heavy) matter content in the theory. Alternatively, we could introduce an additional gauge group to the low energy theory, under which the $B^{\prime}$ and $L^{\prime}$ have differing charges. Such a soft breaking of the $Z_{2}$ flavour symmetry will lead to a small mass splitting between $B^{\prime}$ and $L^{\prime}$, similar to that employed in models of inelastic DM [71] and eXcited DM [72].

To provide a portal to the SM, we can introduce an additional gauge boson, $Z^{\prime}$, under which the states $L^{\prime}$ (and $B^{\prime}$ ) are charged. Provided this has the appropriate mass (through an additional scalar, that we do not specify, gaining a vacuum expectation value) annihilation of $L^{\prime}$ and $\overline{L^{\prime}}$ proceeds to two on-shell $Z^{\prime}$. If the states $N_{1}$ and $N_{2}$ are uncharged under this symmetry, and there are no other lighter hidden sector states to which the $Z^{\prime}$ can decay, then it will be approximately stable. Decay of the $Z^{\prime}$ to the SM can then occur through, for example, a small amount of kinetic mixing with the SM hypercharge U(1). This is particularly well motivated from the perspective of a string theory UV completion. In a IIB model, the hidden sector can arise as a theory on branes at a singularity of the compactification that is geometrically separated from the SM branes. If the distance between the two sectors is large, there are typically no couplings in the low energy theories, except for kinetic mixing which is unsuppressed by such a separation [73]. Alternatively, it is straightforward to introduce an additional pseudoscalar in the theory that couples to the states $L^{\prime}$. This can then act as a portal if it also couples to the SM quarks, leading to a model of the form of section 3.4 .

While in the model presented here the decay of $B^{\prime}$ to $\overline{L^{\prime}}+\cdots$ is mediated through a heavy scalar, there are also well motivated scenarios where this occurs through a heavy vector. In particular, if $B^{\prime}$ and $\bar{L}^{\prime}$ appear in the same multiplet in a (spontaneously broken) GUT theory, this decay may be mediated through a gauge boson charged under the $Z_{2}$

\footnotetext{
${ }^{9}$ As mentioned in appendix $\mathrm{A}$, if $N_{1}$ and $N_{2}$ do not have other interactions, then their masses must generally be $(<\mathcal{O}(\mathrm{eV}))$ to avoid having too large a relic density.
} 


\begin{tabular}{|c|c|c|c|c|}
\hline & constituents & $B^{\prime}$ & $L^{\prime}$ & $B^{\prime}-L^{\prime}$ \\
\hline$B^{\prime}$ & $Q^{\prime} Q^{\prime}$ & 1 & 0 & 1 \\
\hline$L^{\prime}$ & $E^{\prime} E^{\prime}$ & 0 & 1 & -1 \\
\hline$\pi^{\prime}$ & $\bar{Q} Q, \bar{E} E$ & 0 & 0 & 0 \\
\hline
\end{tabular}

Table 2. Lightest bound state singlets of the hidden sector gauge group (plus their anti-partners).

symmetry, analogous to the X, Y bosons that appear in Standard Model GUTs. In this case, the $Z_{2}$ symmetry must also arise as a discrete remnant of part of the hidden sector GUT gauge group, so that the states $B^{\prime}$ and $\bar{L}^{\prime}$ can have differing charges under this.

\subsection{Comments on composite-type models}

Alternatively, a small mass splitting between states can be accomplished in models of composite DM, in analogy to the proton-neutron mass splitting. The phenomenology of such composite models is, while potentially interesting, generally rather complicated, and it is not obvious that all of the necessary constraints can be satisfied in a simple model. Nevertheless, we sketch one such model below, without investigating phenomenological details.

Consider a simple hidden sector with a confining gauge group, say $\mathrm{SU}(2)$, and lefthanded chiral matter content consisting of $Q_{\alpha}^{\prime}$ and $E_{\alpha}^{\prime}$. Suppose the hidden sector $\operatorname{SU}(2)$ runs into strong coupling at about $5 \mathrm{GeV}$, then the theory will confine. As a result calculations of the dynamics in this region are unreliable, hence, we shall simply assume that the lowest mass states which arise as $\mathbf{2} \times \mathbf{2}$ are $E^{\prime} E^{\prime}$ bound states, and identify these with $L^{\prime}$, and the next lightest is the $Q^{\prime} Q^{\prime}$ composite which we call $B^{\prime}$, as given in table 2 . There are also mixed meson states e.g. $\overline{Q^{\prime}} Q^{\prime}, \overline{E^{\prime}} E^{\prime}, \frac{1}{\sqrt{2}}\left(Q^{\prime} E^{\prime} \pm E^{\prime} Q^{\prime}\right)$, which unfortunately complicate the phenomenology.

Again, the theory is assumed to have an exact $B-L-\left(B^{\prime}-L^{\prime}\right)$ symmetry, which leads to approximate $B^{\prime}$ and $L^{\prime}$ symmetries in the low energy effective field theory. The mass splitting between $B^{\prime}$ and $L^{\prime}$ can arise due to radiative corrections, as in the previous 'flavour' example. For instance, suppose the states $Q^{\prime}$ and $E^{\prime}$ are charged under an additional hidden sector gauge group; then the typical size of the $B^{\prime}-L^{\prime}$ mass splittings due to these extra gauge interactions (with coupling constant $g$ ) is parametrically $[74,75]$

$$
\Delta m \sim \frac{g^{2}}{16 \pi^{2}} \Lambda
$$

where $\Lambda$ is the confining scale of the hidden $\mathrm{SU}(2)$.

Further complications arise due to the composite nature of the DM, in particular the annihilation and scattering cross sections can be modified, possibly involving intricate form factors, and it is likely that 'dark pion' $\pi^{\prime}$ exchange will lead to DM self interactions fairly close to current limits. Notably, this potentially also allows for enhancements in the annihilation rates which determine the relic density or indirect detection signals. For some related discussions of composite DM see e.g. [76-80]. Thus, whilst composite models are an 
interesting setting for realising a small mass splitting, given these complications we shall not pursue this scenario in further detail here.

\section{Conclusion}

ADM is a well motivated framework in which to examine proposals of light DM, as if the baryon and DM asymmetries are comparable, then the fact that $\Omega_{\mathrm{DM}} \sim 5 \Omega_{B}$ implies that the DM mass is similar to the proton. Annihilation-like indirect detection signatures are typically not expected if the DM relic density is determined by a particle asymmetry, but can arise in models with more complex hidden sectors. We have outlined a new class of ADM models in which annihilation signals are generated, and presented a number of concrete examples. In particular we discussed a scenario involving two states $B^{\prime}, L^{\prime}$ with comparable relic densities, stabilised by two different approximately conserved quantum numbers. Subsequently, processes which violate these approximate symmetries lead to decays of the heavier state $B^{\prime}$, regenerating the symmetric component of the lighter species $L^{\prime}$. This allows for the prospect of observable indirect detection signals with annihilationlike profiles via $L^{\prime}$-pair annihilation.

One of the principal model-building challenges for producing observable signals in this manner is that, unless the mass splitting between $B^{\prime}$ and $L^{\prime}$ is small, most of the $\overline{L^{\prime}}$ generated via $B^{\prime}$ decays are immediately ejected from the galaxy and do not give rise to annihilation-type signals. The desire for observable annihilation signals constrains the parameter space of the model as discussed in section 3.1 and illustrated in figure 3. Moreover, if the mass splitting is moderate then the symmetric component of $L^{\prime}$ may escape galactic structures with low escape velocities. Thus a distinctive signature of this class of models is that annihilation signals could be observed in our galaxy and conspicuous by their absence in dwarf galaxies. Further, we outlined two scenarios in which such small mass splittings can arise, in the context of broken flavour symmetries, and composite models analogous to the proton-neutron mass splitting.

There is a range of further experimental constraints on these models both from direct and indirect probes, as encapsulated in figures $2,4,6$. In order to evade the strong direct detection bounds we studied two particular scenarios: in the first case annihilations occur on resonance and in the alternative the DM annihilates to pairs of meta-stable hidden sector states. We highlighted the prospect for indirect detection signals in the near future, see for instance figures $2 \& 5$, and commented on tentative signals of DM annihilations near the galactic centre [59-65] in section 3.5.

To conclude, we find that whilst models of low-mass ADM with a symmetric component regenerated by decays can produce observable annihilation signals, and satisfy the various experimental bounds, these models are typically required to possess some specific properties and thus exhibit some predictive features. Several experiments have hinted at the possibility of $\mathrm{DM}$ in the $1-50 \mathrm{GeV}$ range, both direct detection $[81,82]$ and indirect signals of DM annihilation [59-65]. The confirmation of annihilation signals consistent with light DM (possibly with an annihilation cross section different from the thermal freeze-out value) could be an indication of the class of annihilating ADM models proposed here. 


\section{Acknowledgments}

We are grateful to Ulrich Haisch, Dan Hooper, Felix Kahlhoefer, John March-Russell, Chris McCabe, Michele Papucci, and especially Stephen West for useful discussions. EH and RL are funded by STFC Studentships. In addition, RL is grateful for support from the Buckee Scholarship at Merton College, Oxford.

\section{A Rates of $B^{\prime}$ and $L^{\prime}$ violating processes}

In this work, we have assumed that all $B^{\prime}$ and $L^{\prime}$ violating processes apart from the (slow) $B^{\prime} \rightarrow \overline{L^{\prime}}+\cdots$ decay can be neglected. Here, we justify this assumption and estimate the rates of various processes.

Suppose that $B^{\prime}$ and $L^{\prime}$ are good symmetries at low energies, and are only violated at some high scale $M_{B^{\prime}}$. The decay of $B^{\prime}$ is then described by a contact operator $\frac{1}{\Lambda^{k}} B^{\prime} L^{\prime} \mathcal{O}_{k+1}$, where we assume that $B^{\prime}$ and $L^{\prime}$ are fermions, and so $\mathcal{O}_{k+1}$ is a dimension $k+1$ operator. The small mass splitting $\Delta m$ between $B^{\prime}$ and $L^{\prime}$ will result in a phase-space suppression of the decay width, and there will be additional $\Delta m$ factors from any fermionic wavefunctions from $\mathcal{O}_{k+1}$. Dimensionally, a $\operatorname{dim}-(4+k)$ operator will give a total width going parametrically as $(\Delta m)^{2 k+1}$ (or $(\Delta m)^{2 k+3}$ if the $B^{\prime} L^{\prime}$ current gives another $(\Delta m)^{2}$ ). For example, a dimension- 6 operator will, in the former case, give a total width of

$$
\Gamma \sim \frac{(\Delta m)^{5}}{\Lambda^{4}}
$$

In contrast, symmetry-violating annihilations (for instance $B^{\prime}+L^{\prime} \rightarrow \cdots$ ) at high temperatures will not be suppressed, and for a dim- $(4+k)$ operator will have $\sigma \sim \frac{T^{2 k-2}}{\Lambda^{2 k}}$. Hence, in the early universe, the rate of such interactions will be $n_{L^{\prime}}\langle\sigma v\rangle \sim \frac{T^{2 k+1}}{\Lambda^{2 k}}$. So, for $T$ much larger than $\Delta m$, there is the possibility that these will be fast enough to affect the asymmetries in $B^{\prime}$ and $L^{\prime}$ (if these are established above that temperature).

Specifically, since $B^{\prime}-L^{\prime}$ is a good symmetry (ignoring processes that only respect the full $B-L-\left(B^{\prime}-L^{\prime}\right)$ ), we will have $\eta_{B^{\prime}}-\eta_{L^{\prime}}=$ constant. By symmetry, the Boltzmann equation for the other linear combination $\eta_{B^{\prime}}+\eta_{L^{\prime}}$ is

$$
\frac{d\left(\eta_{B^{\prime}}+\eta_{L^{\prime}}\right)}{d x}=-\lambda(x)\left(\eta_{B^{\prime}}+\eta_{L^{\prime}}\right)
$$

to leading order in the small asymmetries, where $x \propto 1 / T$. So, if $\lambda$ is large enough, the asymmetries will be driven towards $\eta_{B^{\prime}}=-\eta_{L^{\prime}}$, corresponding to a population of $B^{\prime}$ and $\overline{L^{\prime}}$ (or the reverse, depending on the sign of $\eta_{B^{\prime}}-\eta_{L^{\prime}}$ ). This would not permit the scenarios we discussed in the main text, where the asymmetries have the same sign. It may be possible to build models in which $B^{\prime}$ and $\overline{L^{\prime}}$ populations give rise to the decay-followed-byannihilation signals considered in the phenomenological sections of this paper (for example, through $B^{\prime} \rightarrow L^{\prime}+\nu \nu+\cdots$ type decays which conserve $B-L-\left(B^{\prime}-L^{\prime}\right)$ ), but we do not go into any model-building details here. 
To check more quantitatively whether this asymmetry-reversal will be important, we take as an example the model of section 4.1, which has (schematically) the symmetryviolating operator $\frac{1}{\Lambda^{2}}\left(B^{\prime} L^{\prime}\right)\left(N_{1}^{\dagger} N_{2}^{\dagger}\right)$. This leads to a three-body decay, with differential width

$$
d \Gamma=\frac{1}{2 m_{B^{\prime}}}|\mathcal{A}|^{2} d q^{2} \frac{d \Omega_{\mathrm{CM}}}{16 \pi^{2}} \frac{\left|\vec{p}_{\mathrm{CM}}\right|}{m_{B^{\prime}}} \frac{1}{2 \pi} \frac{d \Omega_{N}}{16 \pi^{2}} \frac{\left|\vec{p}_{N}\right|}{\sqrt{q^{2}}},
$$

where $q$ is the total 4-momentum of $N_{1}$ and $N_{2}$, and the subscript- $N$ quantities are in the frame where $q$ is purely timelike. Neglecting the masses of $N_{1}$ and $N_{2}$, and assuming that $\Delta m$ is small, we have $\vec{p}_{\mathrm{CM}}^{2} \approx(\Delta m)^{2}-q^{2}$. Also, $|\mathcal{A}|^{2} \approx \frac{4}{\Lambda^{4}} m_{B^{\prime}}^{2} q^{2}$ (summing over final spins), so overall,

$$
\begin{aligned}
\Gamma & \approx \frac{1}{4 \pi m_{B^{\prime}}} \int_{0}^{(\Delta m)^{2}} d q^{2} \frac{1}{4 \pi} \frac{\sqrt{(\Delta m)^{2}-q^{2}}}{m_{B^{\prime}}} \frac{1}{8 \pi} \frac{4}{\Lambda^{4}} m_{B^{\prime}}^{2} q^{2} \\
& =\frac{1}{(4 \pi)^{3}} \frac{8}{15} \frac{(\Delta m)^{5}}{\Lambda^{4}} .
\end{aligned}
$$

In the early universe, symmetry-violating processes of the form $B^{\prime}+L^{\prime} \rightarrow N_{1}+N_{2}$, $B^{\prime}+N_{1} \rightarrow \overline{L^{\prime}}+N_{1}$, etc. will be active. The associated annihilation cross sections are

$$
\sigma \approx C \frac{1}{8 E^{2}} \frac{1}{8 \pi} \frac{1}{\Lambda^{4}} E^{4}
$$

where $E \gg m_{B^{\prime}}$ is the energy of each particle in the CoM frame, and $C$ is a numerical constant depending on which legs are ingoing and outgoing. Thus, in a thermal bath at temperature $T \gg m_{B^{\prime}}$, we have the thermally averaged cross section [83]

$$
\langle\sigma v\rangle \approx \frac{3}{32 \pi} C \frac{T^{2}}{\Lambda^{4}} \equiv C \sigma_{1} \frac{T^{2}}{T_{1}^{2}},
$$

where $T_{1}$ is some (high) temperature. The Boltzmann equation is then (to leading order) of the form

$$
\frac{1}{a^{3}} \frac{d}{d t}\left[a^{3}\left(\left(n_{B^{\prime}}-n \overline{B^{\prime}}\right)+\left(n_{L^{\prime}}-n_{\overline{L^{\prime}}}\right)\right)\right]=-C^{\prime} n_{N_{1}}(T) \sigma_{1} \frac{T^{2}}{T_{1}^{2}}\left[\left(n_{B^{\prime}}-n_{\overline{B^{\prime}}}\right)+\left(n_{L^{\prime}}-n_{\overline{L^{\prime}}}\right)\right],
$$

where $C^{\prime}$ is a numerical constant obtained from summing over all of the leg orderings, with the appropriate weights (its value is $\mathcal{O}(10)$ ). Converting to conserved variables $Y_{i}=n_{i} / s$, and letting $x=T_{1} / T$, this becomes

$$
\frac{d\left(\eta_{B^{\prime}}+\eta_{L^{\prime}}\right)}{d x}=-\frac{\lambda}{x^{4}} \sqrt{g_{*}(T)} Y_{N_{1}}\left(\eta_{B^{\prime}}+\eta_{L^{\prime}}\right),
$$

with

$$
\lambda \simeq 1.32 \times C^{\prime} T_{1} M_{\mathrm{Pl}} \sigma_{1},
$$

where we have used that during the radiation-dominated era, the Hubble rate is given by $H=\frac{T^{2}}{M_{\mathrm{Pl}}^{*}}$, where $M_{\mathrm{Pl}}^{*}=M_{\mathrm{Pl}} \sqrt{\frac{90}{\pi^{2} g_{*}(T)}}$. So, in terms of the $B^{\prime}$ decay rate $\Gamma$, we find that

$$
\lambda=3 \pi^{3} \sqrt{\frac{5}{2}} C^{\prime} \frac{T_{1}^{3} M_{\mathrm{pl}} \Gamma}{(\Delta m)^{5}} .
$$


So, if $T^{3} \gtrsim 10^{-3} \frac{(\Delta m)^{5}}{\Gamma M_{\mathrm{pl}}}$, then $\eta_{B^{\prime}}+\eta_{L^{\prime}}$ will be suppressed by multiple $e$-folds. Taking some representative values, for $\Gamma \sim 1 / t_{0}$ and $\Delta m \sim 10 \mathrm{MeV}$, we find that this corresponds to $T \gtrsim 3 \mathrm{TeV}$. For the models proposed here to be successfully realised, it is required that the $B^{\prime}$ and $L^{\prime}$ asymmetries are established at some point after the universe has cooled below this temperature.

Further, our assumption that the asymmetries are set before $B^{\prime}, L^{\prime}$ freeze-out from the thermal bath, i.e. roughly before they become non-relativistic, implies a lower limit on $\Delta m$, or equivalently, an upper limit on $\Gamma$. For example, for $\Gamma=1 / t_{\mathrm{CMB}}$ it is required that $\Delta m \gtrsim 7 \mathrm{MeV}$, otherwise symmetry-violating interactions do not decouple until $T \lesssim$ $50 \mathrm{GeV}$. This limit is plotted in figure 4, demonstrating that there is viable parameter for all cosmologically slow decay rates.

There is also the possibility of symmetry-violating interactions at late times, but the number densities then are small enough to make these completely negligible. The most frequent will be those involving a $N$ particle, since (in the simplest case) these decouple from the thermal bath when the symmetry-violating interactions discussed above decouple, forming a dark radiation component (the large difference between $g_{*}$ at the time of decoupling and later means that this does not conflict with constraints on $N_{\text {eff }}$ from BBN and the CMB). The $N$ will have a number density of $\sim n_{\gamma} / 8$, where $n_{\gamma} \approx 400 \mathrm{~cm}^{-3} \approx\left(10^{-10} \mathrm{MeV}\right)^{3}$ is the photon number density today, so the rate of e.g. $B^{\prime}+N_{1} \rightarrow \overline{L^{\prime}}+N_{2}$ interactions will be much smaller than the rate of $B^{\prime} \rightarrow \overline{L^{\prime}}+N_{1}+N_{2}$ decays, since $(\Delta m)^{3} \gg n_{\gamma}$. Note that this scenario is only viable for small enough $N$ masses - if the mass were large enough that early decoupling would give too large a relic density, we would need some other mechanism to reduce the eventual abundance (e.g. annihilation to lighter states).

\section{B $\quad B^{\prime}-L^{\prime}$ mass splitting and $\overline{L^{\prime}}$ injection velocity}

As discussed in section 3.1, if the $B^{\prime} \rightarrow \overline{L^{\prime}}+\cdots$ decay has more than two decay products, then we will obtain a distribution of final velocities for the $\overline{L^{\prime}}$. In particular, a fraction of them will obtain velocities higher than some critical value $v_{c}$ (e.g. the escape velocity of a bound structure). Here, we estimate this fraction, and confirm that for mass splittings $m_{B^{\prime}}-m_{L^{\prime}} \equiv \Delta m$ larger than $m_{L^{\prime}} v_{c}$ it is very close to 1 , assuming that none of the final or intermediate states (other than $\overline{L^{\prime}}$ ) have masses close to $\Delta m$.

In the $B^{\prime} \rightarrow \overline{L^{\prime}}+\cdots$ decay, if the other decay products carry away 4-momentum $q$, the velocity given to $\overline{L^{\prime}}$ corresponds to a Lorentz factor $\gamma$ of

$$
\gamma-1=\frac{(\Delta m)^{2}-q^{2}}{2 m_{B^{\prime}} m_{L^{\prime}}}
$$

so if $\Delta m=m_{B}-m_{L}$ is small, the velocity is

$$
v^{2}=\frac{(\Delta m)^{2}-q^{2}}{m_{L^{\prime}}^{2}}+O\left(\left(\frac{\Delta m}{m_{L^{\prime}}}\right)^{3}\right)+O\left(v^{4}\right) .
$$

For a two-body decay $B \rightarrow \bar{L}+X$, assuming that $m_{X}$ is small compared to $\Delta m$ gives $v \approx \frac{\Delta m}{m_{L^{\prime}}}$. For a higher-multiplicity final state, some fraction of the decays will result in 
$v<v_{c}$, i.e. those for which $q^{2}$ is only just below $(\Delta m)^{2}$. We can write the differential decay width to a $n$-body final state as

$$
\mathrm{d} \Gamma=\frac{(2 \pi)^{4}}{2 M}|\mathcal{A}|^{2} \mathrm{~d} \Phi_{n}\left(P ; p_{1}, \ldots, p_{n}\right),
$$

where $M$ is the mass of the decaying particle, $\mathcal{A}$ is the amplitude for that particular decay, and $\mathrm{d} \Phi_{n}$ is the differential phase space element for initial momentum $P$ and final momenta $p_{1}, \ldots, p_{n}$. Also, we have

$$
\mathrm{d} \Phi_{n}\left(P ; p_{1}, \ldots, p_{n}\right)=(2 \pi)^{3} \mathrm{~d} q^{2} \mathrm{~d} \Phi_{2}\left(P ; p_{1}, q\right) \mathrm{d} \Phi_{n-1}\left(q ; p_{2}, \ldots, p_{n}\right),
$$

splitting the decay into an initial two-body step and then a fragmentation of the second body. In the rest frame of the decaying particle, the two-body phase space element has the form $\mathrm{d} \Phi_{2} \propto \mathrm{d} \Omega \frac{|\vec{p}|}{E}$. From above, for small $\Delta m$ we have $|\vec{p}|^{2} \approx(\Delta m)^{2}-q^{2}$, so writing $q^{2}=(\Delta m)^{2}-\delta q^{2}$, the leading order behaviour of $\mathrm{d} \Phi_{2}$ with $\delta q^{2}$ is $\sqrt{\delta q^{2}}$. Since the mass dimension of $\mathrm{d} \Phi_{n}$ is $2 n-4$, if the total mass of the other decay particles is small compared to $\sqrt{\delta q^{2}}$, then $\mathrm{d} \Phi_{n-1}$ must vary like $\left(\delta q^{2}\right)^{n-3}$. So overall, $\mathrm{d} \Phi_{n}$ will vary as $\left(\delta q^{2}\right)^{n-3 / 2}$.

The phase space volume with $v<v_{c}$ corresponds to that with $\delta q^{2}$ below a critical value, and we want to compare the total width for that volume to the total width overall. If we do not have intermediate states with masses close to $\Delta m$ (heavier states have a roughly constant effect on $|\mathcal{A}|$, while lighter states enhance it towards smaller $q^{2}$ ), then we can obtain an approximate upper bound on the ratio of widths by comparing the phase space volumes. From above, as long as the other final state masses are small compared to $\Delta m$, this will be well approximated by $\left(\frac{\delta q^{2}}{(\Delta m)^{2}}\right)^{n-3 / 2}$.

To take an example, suppose that $m_{B^{\prime}}=20 \mathrm{GeV}, m_{L^{\prime}}=10 \mathrm{GeV}$, and $v_{c}=500 \mathrm{~km} \mathrm{~s}^{-1}$, and that the decay is $B^{\prime} \rightarrow \overline{L^{\prime}}+\phi+\phi$, where the $\phi$ are massless states. Then, $v<v_{c}$ corresponds to $\delta q^{2}<5.6 \times 10^{-2} \mathrm{GeV}^{2}$, which is $1.27 \times 10^{-8}$ of the 3-body phase space, while $\left(\frac{\delta q^{2}}{(\Delta m)^{2}}\right)^{3 / 2}=1.31 \times 10^{-8}$. Since $v^{2} \approx \frac{\delta q^{2}}{m_{L^{\prime}}^{2}}$, in general our volume ratio is $\left(\frac{m v_{c}}{\Delta m}\right)^{2 n-3}$ (for $\Delta m>m v_{c}$; otherwise, the whole of the phase space volume corresponds to a velocity kick $\left.<v_{c}\right)$.

\section{Calculating the Galactic $\overline{L^{\prime}}$ distribution}

Suppose that we have a steady-state distribution of (collisionless) DM particles $B^{\prime}$ in a gravitational potential $\Phi$. A small fraction of these then decay, with the decay products including a particle $\overline{L^{\prime}}$ of only very slightly smaller mass, whose relative velocity is consequently non-relativistic. The problem is to calculate the steady-state distribution of the resulting decay product population.

We could approach this in a brute-force way by sampling from a large number of $B^{\prime}$ starting positions and velocities, sampling from the possible relative $\overline{L^{\prime}}$ velocities, then calculating the resulting $\overline{L^{\prime}}$ orbit and accumulating the time spent at given $\vec{x}$ and $\vec{v}$ in this orbit into the overall $\vec{x}, \vec{v}$ distribution (more sophisticated analyses such as [44] take some variation of this approach). However, we can simplify the problem slightly by assuming that the $B^{\prime}$ distribution, and the gravitational potential, are both spherically symmetric 
(and that the $B^{\prime}$ distribution is non-rotating). Although this ignores various effects, their impact should be minor [45]:

- The evolution of the galactic potential with time: most obviously, particles injected before matter has collapsed into galaxies will not behave as outlined, and may be captured into galaxies later if their velocity is sufficiently low. These will then have the same distribution as the 'parent' $B^{\prime}$ and $L^{\prime}$ particles.

We can put a rough upper bound on this effect by estimating the proportion of the early-emitted $\overline{L^{\prime}}$ that are captured into galaxies. Free-streaming with a velocity $u$ suppresses perturbations on conformal scales with $k>H(t) a(t) / u(t)$, as such particles will escape potential wells. Since Hubble expansion reduces the velocity of non-relativistic particles as $1 / a$, we have $u(t)=u\left(t_{1}\right) a(t) / a\left(t_{1}\right)$, where $t_{1}$ is the time of injection, and $u\left(t_{1}\right)$ is the velocity kick. So, the critical $k$ at the present time is $k_{c}=\frac{H_{0} a\left(t_{1}\right)}{u\left(t_{1}\right)}$. If $k_{c} \lesssim 100 \mathrm{kpc}$, the scale relevant to galaxies, then the injected particles will never have clustered into galaxies. Conversely, only states emitted with $a\left(t_{1}\right) \lesssim H_{0} u\left(t_{1}\right)^{-1} /(100 \mathrm{kpc})$ will cluster. During matter domination, $a \sim t^{2 / 3}$, and since $H_{0} /(100 \mathrm{kpc}) \simeq 7 \mathrm{~km} \mathrm{~s}^{-1}$, it follows that for an $\overline{L^{\prime}}$ decay product to be captured it must be produced prior to

$$
t_{1} \lesssim\left(\frac{7 \mathrm{~km} \mathrm{~s}^{-1}}{u\left(t_{1}\right)}\right)^{3 / 2} t_{0}
$$

Taking example velocity kicks from earlier plots, if $u\left(t_{1}\right) \approx 70 \mathrm{~km} \mathrm{~s}^{-1}$, around $3 \%$ of the $\overline{L^{\prime}}$ emitted cluster in this way, and taking $u\left(t_{1}\right) \approx 220 \mathrm{~km} \mathrm{~s}^{-1}$ gives $\sim 5 \times 10^{-3}$ of the $\overline{L^{\prime}}$ with the 'parent' distribution. From inspection of figure 3 , the modified distribution is always a significantly larger fraction of the original density than those values.

- The shape of the DM halo: $N$-body simulations appear to favour ellipsoidal halos, but with a ratio of longest/shortest axes around 0.6 rather than more extreme values.

- Angular momentum of the DM halo: simulations indicate that the velocity bias due to net rotation is insignificant compared to the velocity dispersion, so should not give a large effect.

- The potential of the galactic disk: there is a degeneracy between the contribution of the halo and the disk to the mass of the inner few parsecs of the galaxy. As a result, in the cases where the velocity kick has most effect (i.e. DM profiles with a central density cusp to be smoothed out) the contribution of the disk is less important.

By the Strong Jeans Theorem [45], the steady state phase-space distribution of a system of collisionless particles moving in a spherical potential can be expressed as $f=f(\varepsilon, \vec{L})$, where $\varepsilon$ is the binding energy and $\vec{L}$ is the angular momentum (both per unit mass). Furthermore, since the $B^{\prime} \rightarrow \overline{L^{\prime}}+\cdots$ decay is spherically symmetric, the distribution of 
the $\overline{L^{\prime}}$ in phase space must be of the form $g=g\left(\varepsilon, L^{2}\right)$. So, if we start out with a distribution function (DF) $f(\vec{x}, \vec{v})=f\left(\varepsilon, L^{2}\right)$ for the $B^{\prime}$, we can derive the 'post-injection' DF

$$
h(\vec{x}, \vec{v})=\int \mathrm{d}^{3} v^{\prime} K\left(\left|v-v^{\prime}\right|\right) f\left(\vec{x}, \vec{v}^{\prime}\right)
$$

for the $\overline{L^{\prime}}$, where $K(\Delta v)$ corresponds to the probability of injecting with a given velocity change. In general, $h$ will not be a steady-state distribution, as can be seen by considering e.g. a high-central-density profile with a cool core, which will be smoothed out by a large velocity boost. However, since $\varepsilon$ and $L^{2}$ are preserved along particle orbits, the number of particles in a volume $\mathrm{d} \varepsilon \mathrm{d} L^{2}$ of $\left(\varepsilon, L^{2}\right)$ space will be the same for $h$ and for the steady-state distribution $g$. Then, since $g$ depends only on $\varepsilon$ and $L^{2}$, we can recover it from the $\mathrm{d} \varepsilon \mathrm{d} L^{2}$ density $p$, via $g \mathrm{~d} V=p \mathrm{~d} \varepsilon \mathrm{d} L^{2}$, where $\mathrm{d} V=\mathrm{d}^{3} x \mathrm{~d}^{3} v$ is phase space volume. Explicitly,

$$
\begin{aligned}
\frac{\mathrm{d} V}{\mathrm{~d} \varepsilon \mathrm{d} L^{2}} & =\int \mathrm{d}^{3} x \int \mathrm{d}^{3} v \delta\left(\varepsilon-\left(\psi-\frac{1}{2} v^{2}\right)\right) \delta\left(L^{2}-r^{2} v_{\perp}^{2}\right) \\
& =2 \pi \int \mathrm{d}^{3} x\left(\frac{v}{\sqrt{r^{2} v^{2}-L^{2}}}\right)_{v^{2}=2(\psi(r)-\varepsilon)},
\end{aligned}
$$

where $v_{\perp}$ is the perpendicular-to-radial velocity, and $\psi(r) \equiv-\Phi(r)$ is the maximum binding energy at $r$. Similarly,

$$
p\left(\varepsilon, L^{2}\right)=\int \mathrm{d}^{3} x \int \mathrm{d}^{3} v f(\vec{x}, \vec{v}) \delta\left(\varepsilon-\left(\psi-\frac{1}{2} v^{2}\right)\right) \delta\left(L^{2}-r^{2} v_{\perp}^{2}\right),
$$

and in particular, if the velocity distribution is everywhere isotropic $(h(\vec{x}, \vec{v})=h(\vec{x},|v|))$, then

$$
p\left(\varepsilon, L^{2}\right)=2 \pi \int \mathrm{d}^{3} x\left(\frac{v h(x, v)}{\sqrt{r^{2} v^{2}-L^{2}}}\right)_{v^{2}=2(\psi(r)-\varepsilon)} .
$$

From $g\left(\varepsilon, L^{2}\right)$, we can find the number density $\rho(r)$ by integrating over the appropriate ranges of $\varepsilon$ and $L^{2}$,

$$
\rho(r)=\int \mathrm{d}^{3} \vec{v} g(r, \vec{v})=\int_{v^{2}<2 \psi(r)} \mathrm{d}^{3} \vec{v} g\left(\psi(r)-v^{2} / 2, r^{2} v^{2} \sin ^{2} \theta\right) .
$$

To find a plausible initial DF for $B^{\prime}$, we can assume that the velocity distribution is everywhere isotropic. This implies that the DF depends only on $\varepsilon$ (it is said to be 'ergodic'). Starting from a number density $\rho(r)$ in a potential $\psi(r)$, there is a unique ergodic DF $f(\varepsilon)$ giving $\rho$ [45], described by the Eddington formula

$$
f(\varepsilon)=\frac{1}{\sqrt{8} \pi^{2}} \frac{\mathrm{d}}{\mathrm{d} \varepsilon} \int_{0}^{\varepsilon} \frac{\mathrm{d} \psi}{\sqrt{\varepsilon-\psi}} \frac{\mathrm{d} \rho}{\mathrm{d} \psi} .
$$

For the figures in the main text, we also (for ease of implementation) make the approximation that the $\overline{L^{\prime}}$ distribution $g$ is ergodic, i.e. that it does not depend on $L^{2}$. This can be a poor approximation for large velocities, as can be seen by considering a cool, high-density centre subjected to a large velocity kick, as this results in most trajectories at 
large distances being approximately radial. However, for the not-too-large velocity kicks we consider here (as required for observable annihilation signals), and for the smaller-radius regions we are most concerned about, the approximation is acceptable. For example, taking the $B$ profile to be the NFW $\gamma=1.2$ one considered in figure 8 , and working out the full $\overline{L^{\prime}}$ distribution function $g\left(\varepsilon, L^{2}\right)$ (for $\Delta v=109 \mathrm{~km} \mathrm{~s}^{-1}$ ) at some representative values, we can estimate that the error in $\rho$ at $r=10 r_{\text {deg }}$ (in the notation of figure 8) will be at most a few percent.

Open Access. This article is distributed under the terms of the Creative Commons Attribution License (CC-BY 4.0), which permits any use, distribution and reproduction in any medium, provided the original author(s) and source are credited.

\section{References}

[1] K. Petraki and R.R. Volkas, Review of asymmetric dark matter, Int. J. Mod. Phys. A 28 (2013) 1330028 [arXiv:1305.4939] [INSPIRE].

[2] H. Davoudiasl and R.N. Mohapatra, On relating the genesis of cosmic baryons and dark matter, New J. Phys. 14 (2012) 095011 [arXiv:1203.1247] [INSPIRE].

[3] K.M. Zurek, Asymmetric dark matter: theories, signatures and constraints, Phys. Rept. 537 (2014) 91 [arXiv:1308.0338] [inSPIRE].

[4] R. Kitano, H. Murayama and M. Ratz, Unified origin of baryons and dark matter, Phys. Lett. B 669 (2008) 145 [arXiv:0807.4313] [InSPIRE].

[5] L.J. Hall, J. March-Russell and S.M. West, A unified theory of matter genesis: asymmetric freeze-in, arXiv:1010.0245 [INSPIRE].

[6] H. Davoudiasl, D.E. Morrissey, K. Sigurdson and S. Tulin, Hylogenesis: a unified origin for baryonic visible matter and antibaryonic dark matter, Phys. Rev. Lett. 105 (2010) 211304 [arXiv: 1008.2399] [INSPIRE].

[7] J. March-Russell and M. McCullough, Asymmetric dark matter via spontaneous co-genesis, JCAP 03 (2012) 019 [arXiv: 1106.4319] [INSPIRE].

[8] W.-Z. Feng and P. Nath, Cogenesis in a universe with vanishing $B-L$ within a gauged $\mathrm{U}(1)_{x}$ extension, Phys. Lett. B 731 (2014) 43 [arXiv:1312.1334] [INSPIRE].

[9] C. Boehm, T. Delahaye and J. Silk, Can the morphology of gamma-ray emission distinguish annihilating from decaying dark matter?, Phys. Rev. Lett. 105 (2010) 221301 [arXiv: 1003.1225] [INSPIRE].

[10] W. Buchmüller and M. Garny, Decaying vs annihilating dark matter in light of a tentative gamma-ray line, JCAP 08 (2012) 035 [arXiv:1206.7056] [INSPIRE].

[11] Y. Zhao and K.M. Zurek, Indirect detection signatures for the origin of asymmetric dark matter, arXiv:1401.7664 [INSPIRE].

[12] M.R. Buckley and S. Profumo, Regenerating a symmetry in asymmetric dark matter, Phys. Rev. Lett. 108 (2012) 011301 [arXiv:1109.2164] [INSPIRE].

[13] M. Cirelli, P. Panci, G. Servant and G. Zaharijas, Consequences of DM/antiDM oscillations for asymmetric WIMP dark matter, JCAP 03 (2012) 015 [arXiv:1110.3809] [INSPIRE]. 
[14] S. Tulin, H.-B. Yu and K.M. Zurek, Oscillating asymmetric dark matter, JCAP 05 (2012) 013 [arXiv: 1202.0283] [INSPIRE].

[15] N. Okada and O. Seto, Originally asymmetric dark matter, Phys. Rev. D 86 (2012) 063525 [arXiv: 1205.2844] [INSPIRE].

[16] A. Falkowski, J.T. Ruderman and T. Volansky, Asymmetric dark matter from leptogenesis, JHEP 05 (2011) 106 [arXiv:1101.4936] [INSPIRE].

[17] S.D. Thomas, Baryons and dark matter from the late decay of a supersymmetric condensate, Phys. Lett. B 356 (1995) 256 [hep-ph/9506274] [INSPIRE].

[18] F. D'Eramo, L. Fei and J. Thaler, Dark matter assimilation into the baryon asymmetry, JCAP 03 (2012) 010 [arXiv:1111.5615] [inSPIRE].

[19] J. Unwin, Exodus: hidden origin of dark matter and baryons, JHEP 06 (2013) 090 [arXiv: 1212.1425] [INSPIRE].

[20] P.J. Fox, R. Harnik, J. Kopp and Y. Tsai, LEP shines light on dark matter, Phys. Rev. D 84 (2011) 014028 [arXiv: 1103.0240] [INSPIRE].

[21] M.R. Buckley, Asymmetric dark matter and effective operators, Phys. Rev. D 84 (2011) 043510 [arXiv: 1104.1429] [INSPIRE].

[22] J. March-Russell, J. Unwin and S.M. West, Closing in on asymmetric dark matter I: model independent limits for interactions with quarks, JHEP 08 (2012) 029 [arXiv:1203.4854] [INSPIRE].

[23] G. Bélanger, A. Goudelis, J.-C. Park and A. Pukhov, Isospin-violating dark matter from a double portal, JCAP 02 (2014) 020 [arXiv:1311.0022] [INSPIRE].

[24] E. Del Nobile, G.B. Gelmini, P. Gondolo and J.-H. Huh, Update on light WIMP limits: LUX, lite and light, JCAP 03 (2014) 014 [arXiv:1311.4247] [INSPIRE].

[25] P.J. Fox, G. Jung, P. Sorensen and N. Weiner, Dark matter in light of LUX, Phys. Rev. D 89 (2014) 103526 [arXiv:1401.0216] [InSPIRE].

[26] M.I. Gresham and K.M. Zurek, Light dark matter anomalies after LUX, Phys. Rev. D 89 (2014) 016017 [arXiv: 1311.2082] [INSPIRE].

[27] R.C. Cotta, J.L. Hewett, M.P. Le and T.G. Rizzo, Bounds on dark matter interactions with electroweak gauge bosons, Phys. Rev. D 88 (2013) 116009 [arXiv:1210.0525] [INSPIRE].

[28] I.M. Shoemaker, Constraints on dark matter protohalos in effective theories and neutrinophilic dark matter, Phys. Dark Univ. 2 (2013) 157 [arXiv:1305.1936] [INSPIRE].

[29] D.P. Finkbeiner, S. Galli, T. Lin and T.R. Slatyer, Searching for dark matter in the CMB: a compact parameterization of energy injection from new physics, Phys. Rev. D 85 (2012) 043522 [arXiv:1109.6322] [InSPIRE].

[30] X.-L. Chen and M. Kamionkowski, Particle decays during the cosmic dark ages, Phys. Rev. D 70 (2004) 043502 [astro-ph/0310473] [INSPIRE].

[31] M. Kamionkowski and A. Kosowsky, The cosmic microwave background and particle physics, Ann. Rev. Nucl. Part. Sci. 49 (1999) 77 [astro-ph/9904108] [INSPIRE].

[32] M.S. Madhavacheril, N. Sehgal and T.R. Slatyer, Current dark matter annihilation constraints from CMB and low-redshift data, Phys. Rev. D 89 (2014) 103508 [arXiv:1310.3815] [INSPIRE]. 
[33] J. Shelton and K.M. Zurek, Darkogenesis: a baryon asymmetry from the dark matter sector, Phys. Rev. D 82 (2010) 123512 [arXiv: 1008.1997] [InSPIRE].

[34] N. Haba and S. Matsumoto, Baryogenesis from dark sector, Prog. Theor. Phys. 125 (2011) 1311 [arXiv:1008.2487] [InSPIRE].

[35] M.R. Buckley and L. Randall, Xogenesis, JHEP 09 (2011) 009 [arXiv:1009.0270] [INSPIRE].

[36] P. Nath and P. Fileviez Perez, Proton stability in grand unified theories, in strings and in branes, Phys. Rept. 441 (2007) 191 [hep-ph/0601023] [INSPIRE].

[37] LAT collaboration, M. Ackermann et al., Constraints on the galactic halo dark matter from Fermi-LAT diffuse measurements, Astrophys. J. 761 (2012) 91 [arXiv:1205.6474] [INSPIRE].

[38] I.Z. Rothstein, T. Schwetz and J. Zupan, Phenomenology of dark matter annihilation into a long-lived intermediate state, JCAP 07 (2009) 018 [arXiv:0903.3116] [INSPIRE].

[39] J. Mardon, Y. Nomura, D. Stolarski and J. Thaler, Dark matter signals from cascade annihilations, JCAP 05 (2009) 016 [arXiv:0901.2926] [INSPIRE].

[40] S. Tulin, H.-B. Yu and K.M. Zurek, Beyond collisionless dark matter: particle physics dynamics for dark matter halo structure, Phys. Rev. D 87 (2013) 115007 [arXiv:1302.3898] [INSPIRE].

[41] J.L. Feng, M. Kaplinghat and H.-B. Yu, Halo shape and relic density exclusions of Sommerfeld-enhanced dark matter explanations of cosmic ray excesses,

Phys. Rev. Lett. 104 (2010) 151301 [arXiv:0911.0422] [INSPIRE].

[42] T. Lin, H.-B. Yu and K.M. Zurek, On symmetric and asymmetric light dark matter, Phys. Rev. D 85 (2012) 063503 [arXiv:1111.0293] [InSPIRE].

[43] M. Kaplinghat, R.E. Keeley, T. Linden and H.-B. Yu, Tying dark matter to baryons with self-interactions, arXiv:1311.6524 [INSPIRE].

[44] A.H.G. Peter, C.E. Moody and M. Kamionkowski, Dark-matter decays and self-gravitating halos, Phys. Rev. D 81 (2010) 103501 [arXiv:1003.0419] [INSPIRE].

[45] J. Binney and S. Tremaine, Galactic dynamics, 2nd edition, Princeton University Press, Princeton U.S.A. (2008).

[46] R. Essig, E. Kuflik, S.D. McDermott, T. Volansky and K.M. Zurek, Constraining light dark matter with diffuse X-ray and gamma-ray observations, JHEP 11 (2013) 193 [arXiv: 1309.4091] [INSPIRE].

[47] N.F. Bell, A.J. Galea and K. Petraki, Lifetime constraints for late dark matter decay, Phys. Rev. D 82 (2010) 023514 [arXiv: 1004.1008] [INSPIRE].

[48] A.H.G. Peter and A.J. Benson, Dark-matter decays and Milky Way satellite galaxies, Phys. Rev. D 82 (2010) 123521 [arXiv:1009.1912] [inSPIRE].

[49] M.-Y. Wang and A.R. Zentner, Effects of unstable dark matter on large-scale structure and constraints from future surveys, Phys. Rev. D 85 (2012) 043514 [arXiv:1201.2426] [INSPIRE].

[50] M.-Y. Wang, R.A.C. Croft, A.H.G. Peter, A.R. Zentner and C.W. Purcell, Lyman- $\alpha$ forest constraints on decaying dark matter, Phys. Rev. D 88 (2013) 123515 [arXiv:1309.7354] [INSPIRE].

[51] N. Fornengo, L. Maccione and A. Vittino, Constraints on particle dark matter from cosmic-ray antiprotons, JCAP 04 (2014) 003 [arXiv: 1312.3579] [INSPIRE]. 
[52] C. Evoli, I. Cholis, D. Grasso, L. Maccione and P. Ullio, Antiprotons from dark matter annihilation in the galaxy: astrophysical uncertainties, Phys. Rev. D 85 (2012) 123511 [arXiv:1108.0664] [INSPIRE].

[53] L. Bergström, T. Bringmann, I. Cholis, D. Hooper and C. Weniger, New limits on dark matter annihilation from AMS cosmic ray positron data, Phys. Rev. Lett. 111 (2013) 171101 [arXiv: 1306.3983] [INSPIRE].

[54] A.R. Zentner, High-energy neutrinos from dark matter particle self-capture within the Sun, Phys. Rev. D 80 (2009) 063501 [arXiv:0907.3448] [InSPIRE].

[55] I.F.M. Albuquerque, C. Pérez de Los Heros and D.S. Robertson, Constraints on self interacting dark matter from IceCube results, JCAP 02 (2014) 047 [arXiv:1312.0797] [INSPIRE].

[56] M. Pospelov, A. Ritz and M.B. Voloshin, Secluded WIMP dark matter, Phys. Lett. B 662 (2008) 53 [arXiv:0711.4866] [INSPIRE].

[57] D.N. Spergel and P.J. Steinhardt, Observational evidence for selfinteracting cold dark matter, Phys. Rev. Lett. 84 (2000) 3760 [astro-ph/9909386] [INSPIRE].

[58] G. Bélanger, F. Boudjema, A. Pukhov and A. Semenov, MicrOMEGAs: a program for calculating the relic density in the MSSM, Comput. Phys. Commun. 149 (2002) 103 [hep-ph/0112278] [INSPIRE].

[59] D. Hooper and T.R. Slatyer, Two emission mechanisms in the Fermi bubbles: a possible signal of annihilating dark matter, Phys. Dark Univ. 2 (2013) 118 [arXiv:1302.6589] [INSPIRE].

[60] T. Daylan et al., The characterization of the gamma-ray signal from the central Milky Way: a compelling case for annihilating dark matter, arXiv:1402.6703 [INSPIRE].

[61] D. Hooper, I. Cholis, T. Linden, J. Siegal-Gaskins and T. Slatyer, Millisecond pulsars cannot account for the inner galaxy's GeV excess, Phys. Rev. D 88 (2013) 083009 [arXiv: 1305.0830] [INSPIRE].

[62] K.N. Abazajian and M. Kaplinghat, Detection of a gamma-ray source in the galactic center consistent with extended emission from dark matter annihilation and concentrated astrophysical emission, Phys. Rev. D 86 (2012) 083511 [arXiv:1207.6047] [INSPIRE].

[63] D. Hooper and T. Linden, On the origin of the gamma rays from the galactic center, Phys. Rev. D 84 (2011) 123005 [arXiv:1110.0006] [InSPIRE].

[64] D. Hooper and L. Goodenough, Dark matter annihilation in the galactic center as seen by the Fermi Gamma Ray Space Telescope, Phys. Lett. B 697 (2011) 412 [arXiv:1010.2752] [INSPIRE].

[65] K.N. Abazajian, N. Canac, S. Horiuchi and M. Kaplinghat, Astrophysical and dark matter interpretations of extended gamma ray emission from the galactic center, arXiv:1402.4090 [INSPIRE].

[66] C. Gordon and O. Macias, Dark matter and pulsar model constraints from galactic center Fermi-LAT gamma ray observations, Phys. Rev. D 88 (2013) 083521 [arXiv:1306.5725] [INSPIRE].

[67] W. Dehnen and A. King, Probing dark matter with X-ray binaries, Mon. Not. Roy. Astron. Soc. Lett. 367 (2006) L29 [astro-ph/0512106] [INSPIRE]. 
[68] M.G. Walker et al., Velocity dispersion profiles of seven dwarf spheroidal galaxies, arXiv:0708.0010 [INSPIRE].

[69] A. Charbonnier et al., Dark matter profiles and annihilation in dwarf spheroidal galaxies: prospectives for present and future gamma-ray observatories - I. The classical dwarf spheroidal galaxies, Mon. Not. Roy. Astron. Soc. 418 (2011) 1526 [arXiv:1104.0412] [INSPIRE].

[70] C. Boehm, M.J. Dolan, C. McCabe, M. Spannowsky and C.J. Wallace, Extended gamma-ray emission from coy dark matter, JCAP 05 (2014) 009 [arXiv: 1401.6458] [INSPIRE].

[71] D. Tucker-Smith and N. Weiner, Inelastic dark matter, Phys. Rev. D 64 (2001) 043502 [hep-ph/0101138] [INSPIRE].

[72] D.P. Finkbeiner and N. Weiner, Exciting dark matter and the INTEGRAL/SPI $511 \mathrm{keV}$ signal, Phys. Rev. D 76 (2007) 083519 [astro-ph/0702587] [INSPIRE].

[73] S.A. Abel, M.D. Goodsell, J. Jaeckel, V.V. Khoze and A. Ringwald, Kinetic mixing of the photon with hidden $\mathrm{U}(1)$ s in string phenomenology, JHEP 07 (2008) 124 [arXiv:0803.1449] [INSPIRE].

[74] N. Arkani-Hamed, D.P. Finkbeiner, T.R. Slatyer and N. Weiner, A theory of dark matter, Phys. Rev. D 79 (2009) 015014 [arXiv: 0810.0713] [inSPIRE].

[75] M. Baumgart, C. Cheung, J.T. Ruderman, L.-T. Wang and I. Yavin, Non-Abelian dark sectors and their collider signatures, JHEP 04 (2009) 014 [arXiv:0901.0283] [INSPIRE].

[76] J.D. March-Russell and S.M. West, WIMPonium and boost factors for indirect dark matter detection, Phys. Lett. B 676 (2009) 133 [arXiv:0812.0559] [INSPIRE].

[77] W. Shepherd, T.M.P. Tait and G. Zaharijas, Bound states of weakly interacting dark matter, Phys. Rev. D 79 (2009) 055022 [arXiv:0901.2125] [InSPIRE].

[78] D.E. Kaplan, G.Z. Krnjaic, K.R. Rehermann and C.M. Wells, Atomic dark matter, JCAP 05 (2010) 021 [arXiv: 0909.0753] [INSPIRE].

[79] J.M. Cline, Z. Liu, G. Moore and W. Xue, Scattering properties of dark atoms and molecules, Phys. Rev. D 89 (2014) 043514 [arXiv:1311.6468] [InSPIRE].

[80] J.M. Cline, Z. Liu, G. Moore and W. Xue, Composite strongly interacting dark matter, arXiv:1312.3325 [INSPIRE].

[81] CDMS collaboration, R. Agnese et al., Silicon detector dark matter results from the final exposure of CDMS II, Phys. Rev. Lett. 111 (2013) 251301 [arXiv:1304.4279] [INSPIRE].

[82] CoGeNT collaboration, C.E. Aalseth et al., Results from a search for light-mass dark matter with a P-type point contact germanium detector, Phys. Rev. Lett. 106 (2011) 131301 [arXiv: 1002.4703] [INSPIRE].

[83] P. Gondolo and G. Gelmini, Cosmic abundances of stable particles: improved analysis, Nucl. Phys. B 360 (1991) 145 [InSPIRE]. 